\title{
Existence of cube terms in finite algebras
}

\author{
Alexandr Kazda and Dmitriy Zhuk
}

\begin{abstract}
We study the problem of whether a given finite algebra with finitely many basic operations contains a cube term; we give both structural and algorithmic results. We show that if such an algebra has a cube term then it has a cube term of dimension at most $N$, where the number $N$ depends on the arities of basic operations of the algebra and the size of the basic set. For finite idempotent algebras we give a tight bound on $N$ that, in the special case of algebras with more than $\left(\begin{array}{c}|A| \\ 2\end{array}\right)$ basic operations, improves an earlier result of K. Kearnes and A. Szendrei. On the algorithmic side, we show that deciding the existence of cube terms is in $\mathrm{P}$ for idempotent algebras and in EXPTIME in general.

Since an algebra contains a $k$-ary near unanimity operation if and only if it contains a $k$-dimensional cube term and generates a congruence distributive variety, our algorithm also lets us decide whether a given finite algebra has a near unanimity operation.
\end{abstract}

Mathematics Subject Classification. 08B05, 08A70.

Keywords. cube term, cube term blocker, near unanimity, few subpowers, algorithm, idempotent algebra.

\section{Introduction}

Our goal in this paper is to explore the conditions for the existence of a cube term in finite a algebra (with finitely many basic operations). A finite algebra has few subalgebras of powers if and only if it has a cube term (equivalently, an edge term or a parallelogram term) of some dimension. See [3] and [1] for an introduction to these terms, and [10] for the application to the Constraint Satisfaction Problem.

How to efficiently decide if a given finite algebra has a cube term? This question has practical significance: When coming up with hypotheses about

The first author was supported by the PRIMUS/SCI/12 and UNCE/SCI/22 projects of the Charles University and by the the Czech Science Foundation project GA CR 18-20123S. The second author was supported by Russian Foundation for Basic Research (grant 1901-00200). Our thanks go to Ralph Freese who has, with amazing speed, implemented Algorithm 1 in UAcalc. 
few subpowers, one might want to quickly know if a candidate for a counterexample actually has few subpowers. Deciding existence of various operations is also of interest in computational universal algebra (e.g. in the software package UAcalc [6]).

Conveniently, deciding whether an algebra $\mathbf{A}$ has a cube term goes a long way towards telling us whether $\mathbf{A}$ also has a near unanimity operation as $\mathbf{A}$ has a near unanimity operation if and only if $\mathbf{A}$ has a cube term and A generates a congruence distributive variety.

Since we present an EXPTIME algorithm for the former property and deciding the latter property is EXPTIME-complete for general algebras [7], we can place the problem of deciding if an algebra has a near unanimity term into the class EXPTIME. This is a significant improvement over the previous result [15] that the problem is algorithmically decidable.

Besides the general case, we also look at deciding the existence of cube terms for idempotent algebras. Our Algorithm 1 shows how to do this in polynomial time. This improves a previous algorithm given in [14, Corollary 3.6]. Previously, a polynomial time algorithm was known for deciding cube terms in idempotent algebras when the support set $A$ was fixed (from the proof of [14, Corollary 3.6] it in fact follows that the problem is fixed parameter tractable when parameterized by $|A|)$. Our Algorithm 1 improves this result by making the running time depend polynomially on $|A|$.

Finally, a problem related to deciding cube terms is to decide if a given algebra has a cube term of a given fixed dimension. Horowitz investigates this problem [9] and, among other things, gives a polynomial time to decide whether an idempotent algebra has a cube term of a given fixed dimension. However, the local to global method from [9] does not easily extend to the situation when we want a cube term of any dimension.

Before we begin, we would like to point out that the part of our paper devoted to idempotent algebras (Section 4) has a significant overlap with the results in 12 by Keith Kearnes and Agnes Szendrei. To be specific, [12, Theorem 4.1] is a version of our Theorem 20. Both our Theorem 20] and [12, Theorem 4.1] talk about idempotent algebras with finitely many basic operations. Unlike Theorem 4.1, our Theorem 20 only applies to finite algebras. However, on its home ground Theorem 20 sometimes offers a better upper bound on the dimension of a cube term; this happens for idempotent algebras with more than $\left(\begin{array}{c}|A| \\ 2\end{array}\right)$ basic operations. This makes Theorem20 better suited for examining finite algebras. In fact, we use it to obtain a bound on the minimum dimension of a cube term that depends quadratically on $|A|$ and linearly on the maximum arity of a basic operation of $\mathbf{A}$ (Corollary 21).

Another topic our paper shares with [12] is the construction of algebras proving that the bound on the dimension of a cube term from Theorem 20 . (or [12, Theorem 4.1]) is tight; this is Theorem 22 here and Example 4.4 in 12. While the outcome is similar, our construction is novel in that it works for any (finite, greater than 2) size of the base set. 
When constructing the proof of Theorem [20, we had heard the statement of Theorem 4.1 in [12, thus priority for the result belongs to Keith Kearnes and Agnes Szendrei. It also turns out that our methods for idempotent algebras resemble those of [12] (in particular, our "chipped cubes" generalize the "crosses" of Keith Kearnes and Agnes Szendrei). However, we produced the proof of Theorem 20 on our own as we only knew the statement, not the proof of [12, Theorem 4.1] when discovering our proof.

We include a full proof of Theorem 20 in this paper because it illustrates the ideas we later develop for the non-idempotent case and also because Theorem 20 naturally leads to Theorem 22 which shows that the bounds on cube term dimension of Theorem 20 are tight for all applicable sizes of the base set of the algebra in question, improving the state of the art.

\section{Preliminaries}

We will be using basic notations such as operations, algebra of a given signature, clone of operations of an algebra, product of algebras, subuniverse, subuniverse generated by a set, variety and identity (equation) as is usual in universal algebra. See, e.g, [5, 2].

We will spend much time and effort designing and examining tuples. A tuple on $A$ of arity $n$ (or $n$-tuple on $A$ ) is a sequence of $n$ members of the set $A$. If we want to emphasize that an object is a tuple we print it in bold: a. The set of all $n$-tuples on $A$ will be denoted by $A^{n}$. If $\mathbf{a} \in A^{n}$ and $i \in \mathbb{N}$, we denote the $i$-th entry of a by $a_{i}$ or sometimes by $(\mathbf{a})_{i}$. On the other hand, indices without parentheses and in bold shall refer to a particular member of a sequence of tuples, so e.g. $\mathbf{a}_{3}$ is the third tuple from some sequence, not the third entry of $\mathbf{a}$. If confusion is unlikely, we will write the tuple $\left(a_{1}, a_{2}, \ldots, a_{n}\right)$ in a more compact form as $a_{1} a_{2} \ldots a_{n}$.

When $\mathbf{a} \in A^{n}, \mathbf{b} \in A^{k}$ are tuples, we will denote by $\mathbf{a b}$ their concatenation, i.e. the tuple $a_{1} a_{2} \ldots a_{n} b_{1} b_{2} \ldots b_{k} \in A^{n+k}$.

If $i \leq j$ are positive integers and $\mathbf{a}$ is an $n$-tuple, then by $\mathbf{a}_{[i, j]}$ we mean the $(j-i+1)$-tuple $a_{i} a_{i+1} \ldots a_{j}$.

If $a \in A$ and $k \in \mathbb{N}$ then $a^{\mathbf{k}}$ is the $k$-tuple whose all entries are $a$ (the boldface $k$ signals that we are turning $a$ into a tuple), i.e. $a^{\mathbf{k}}=a a \ldots a$. If $\mathbf{a}=a_{1}^{\mathbf{n}_{1}} a_{2}^{\mathbf{n}_{2}} \ldots a_{k}^{\mathbf{n}_{\mathbf{k}}}$ for some $a_{1}, \ldots, a_{k} \in A$, we will call the interval $B_{i}=$ $\left\{j: n_{1}+\cdots+n_{i-1}<j \leq n_{1}+\cdots+n_{i}\right\}$ the $i$-th block of $\mathbf{a}$. The partition into blocks can be ambiguous if e.g. $a_{1}=a_{2}$, but this will not be an issue as we will usually fix the partition in advance. A careful reader might have noticed that we have overloaded e.g. $a_{2}$ to mean both the second element and the $\left(n_{1}+1\right)$-th element of $\mathbf{a}$. We did this to keep our notation short; when a is broken into obvious blocks then $a_{2}$ always stands for the element forming up the second block of $\mathbf{a}$.

Finally, if $A$ is a (finite) set then $\operatorname{seq}(A)$ is the $|A|$-tuple that lists all elements of $A$ in a fixed order (for example, $\operatorname{seq}(\{3,1,4\})=(1,3,4)$ ). We will denote the set $\{1,2, \ldots, n\}$ by $[n]$. 
The following way to combine tuples, introduced in [3], will be useful when talking about cube terms and blockers:

Definition 1. Let $n \in \mathbb{N}, \mathbf{a}, \mathbf{b} \in A^{n}$ be two tuples. We then define for each $I \subseteq[n]$ the $n$-tuple $\chi_{I}(\mathbf{a}, \mathbf{b})$ by

$$
\left(\chi_{I}(\mathbf{a}, \mathbf{b})\right)_{i}= \begin{cases}b_{i} & \text { if } i \in I \\ a_{i} & \text { if } i \notin I .\end{cases}
$$

In particular, we will often consider the matrix $\left(\chi_{I}(\mathbf{a}, \mathbf{b}): I \neq \emptyset\right)$. If $\mathbf{a}, \mathbf{b} \in A^{n}$, then $\left(\chi_{I}(\mathbf{a}, \mathbf{b}): I \neq \emptyset\right)$ is an $n \times\left(2^{n}-1\right)$ matrix whose $i$-th column is $\chi_{I}(\mathbf{a}, \mathbf{b})$ where $I$ is the $i$-th nonempty subset of $[n]$ (ordered in some fixed way).

We will often apply a term operation to a matrix. Let $\mathbf{A}$ be an algebra and let $t$ be an $m$-ary operation of $\mathbf{A}$. If $M$ is an $n \times m$ matrix, then $t(M)$ is the $n$-tuple that we obtain by applying $t$ on the rows of $M$.

For $E \subseteq A^{n}$, we will denote by $\operatorname{Sg}_{\mathbf{A}^{n}}(E)$ the subuniverse of $\mathbf{A}^{n}$ generated by $E$. If we want to emphasize the dimension and the algebra we are talking about, we will write $\operatorname{Sg}_{\mathbf{A}^{n}}(E)$. To simplify notation, we will often omit curly brackets in the argument of $\mathrm{Sg}$, writing e.g. $\operatorname{Sg}(u, v)$ instead of $\operatorname{Sg}(\{u, v\})$.

The algebra $\mathbf{A}$ is idempotent if the identity $t(a, a, \ldots, a)=a$ holds for each term operation $t$ of $\mathbf{A}$ and all $a \in A$ (this is equivalent to demanding that the identity holds for each of A's basic operations).

Our situation in the rest of the paper is that we are given a finite algebra A described by a list of its elements and the tables of its (finitely many) basic operations, and we want to decide if there is a cube term in A. Occasionally, we shall need to distinguish the number of elements of $\mathbf{A}$, denoted by $|A|$, from the total size of the input which includes the list of elements plus a table of size $|A|^{r}$ for each basic operation of $\mathbf{A}$ of arity $r$. We denote the total size of A's description by $|\mathbf{A}|$.

Given a set $S \subseteq A$, we can find the subalgebra of $\mathbf{A}$ generated by $S$ in time $O(m|\mathbf{A}|)$, where $m$ is the maximum arity of a basic operation of $\mathbf{A}$. The algorithm works by generating a sequence $S=S_{0} \subsetneq S_{1} \subsetneq S_{2} \subsetneq \ldots$ of subsets of $A$ that terminates with $\operatorname{Sg}(S)$. We obtain $S_{i+1}$ from $S_{i}$ by applying all the basic operations of $\mathbf{A}$ to tuples from $S_{i}$ that contain at least one element outside of $S_{i-1}$ (this last condition ensures that we handle each tuple at most once for each basic operation; in the $i=0$ step, we let $S_{-1}=\emptyset$ ). We do not claim authorship of this algorithm; it was previously mentioned in [7].

Definition 2. Let $\mathbf{A}$ be an algebra. A relation $R \subseteq A^{n}$ is compatible with $\mathbf{A}$ (also called $\mathbf{A}$-invariant or admissible in the literature) if $R$ is a subuniverse of $\mathbf{A}^{n}$. In other words, $R \leq \mathbf{A}^{n}$ if for every $m$-ary basic operation $f$ of $\mathbf{A}$ the operation $f$ extended to $A^{n}$ maps $R$ into itself: For every $\mathbf{r}_{\mathbf{1}}, \ldots, \mathbf{r}_{\mathbf{m}} \in R$ we have $f(M) \in R$ where $M$ is the $n \times m$ matrix whose $j$-th column is $\mathbf{r}_{\mathbf{j}}$.

The following proposition is easily proved from the definition of relations compatible with an algebra. We note that this proposition is part of a larger 
theory of Galois correspondence between clones of operations and relational clones (sets of relations closed under primitive positive definitions) [4, 8].

Proposition 3. Let $R \leq \mathbf{A}^{n}, S \leq \mathbf{A}^{m}$ be relations compatible with $\mathbf{A}$. Then the following relations are also compatible with $\mathbf{A}$ :

(1) The unary relations $A$ and $\emptyset$,

(2) the projection of $R$ to any subset of $[n]$,

(3) the relation $R \times S$,

(4) if $n=m$, the relation $R \cap S$, and

(5) the unary relation $\{(a)\}$, where $a \in \mathbf{A}$, if $\mathbf{A}$ is idempotent.

One application of Proposition 3 that we will use is that if $R$ is an $n$-ary relation compatible with an idempotent algebra $\mathbf{A}$ and $a$ is an element of $A$, then the relation $S=\left\{\left(s_{1}, \ldots, s_{n-1}\right):\left(a, s_{1}, \ldots, s_{n-1}\right) \in R\right\}$ we get from $R$ by "fixing the first coordinate to $a$ and projecting it out" is also compatible with A.

A $\left(2^{d}-1\right)$-ary operation $t$ on $A$ is called a cube operation of dimension $d$ if for all $\mathbf{a}, \mathbf{b} \in A^{d}$ we have

$$
t\left(\chi_{I}(\mathbf{a}, \mathbf{b}): \emptyset \neq I \subseteq[d]\right)=\mathbf{a} .
$$

The reason for this name is that if we view $2^{I}$ as a $d$-dimensional cube, then the cube term will, given a cube with one missing vertex, fill in the empty spot.

A $(d+1)$-ary operation $u$ is called an edge operation of dimension $d$ (or just a $d$-edge operation) if for any $\mathbf{a}, \mathbf{b} \in A^{d}$ we have

$$
u\left(\chi_{I}(\mathbf{a}, \mathbf{b}): I=\{1,2\},\{1\},\{2\}, \ldots,\{d\}\right)=\mathbf{a} .
$$

Finally, a $d$-ary operation $n$ is a $d$-ary near unanimity operation if for any $\mathbf{a}, \mathbf{b} \in A^{d}$ we have

$$
n\left(\chi_{I}(\mathbf{a}, \mathbf{b}): I=\{1\},\{2\}, \ldots,\{d\}\right)=\mathbf{a} .
$$

(This is not the usual way to write the near unanimity equalities, but we chose it here to show the similarity between near unanimity and cube/edge operations.)

Note that since all three of the above definitions (cube, edge, and near unanimity operations) require that an equality holds for any $\mathbf{a}, \mathbf{b} \in A^{d}$, we could have also easily rewritten them as systems of identities in two variables. For example, a near unanimity operation is an operation satisfying the following identities for all $x, y \in A$ :

$$
f(y, x, \ldots, x)=f(x, y, x, \ldots, x)=\cdots=f(x, \ldots, x, y)=x .
$$

It is easy to see that a $d$-dimensional edge term implies the existence of a $d$-dimensional cube term. It turns out that one can also prove the converse:

Theorem 4 ([3, Theorem 2.12]). Let $\mathbf{A}$ be an algebra. Then $\mathbf{A}$ has a $d$ dimensional edge term operation if and only if $\mathbf{A}$ has a d-dimensional cube term operation. 
The situation with near unanimity is a bit more complicated since having a $d$-ary near unanimity operation is a strictly stronger condition than having an edge or a cube term. However, it turns out that having a $k$-ary near unanimity operation is equivalent to having a $k$-edge term (or, by Theorem 4, $k$-dimensional cube term) for algebras in congruence distributive varieties (for an earlier result of a similar flavor, see [16, Theorem 3.16]).

Theorem 5 ([3, Theorem 4.4]). For each $k \geq 3$, a variety $\mathcal{V}$ is congruence distributive and has a $k$-edge term $t$ if and only if $\mathcal{V}$ has a $k$-ary near unanimity term.

Thus any bound on the minimal dimension of a cube term is also a bound on the minimal arity of a near unanimity operation.

As noted in the Introduction, deciding the existence of a near unanimity term reduces to deciding the existence of a cube term: By Theorem 5, an algebra $\mathbf{A}$ has a near unanimity term if and only if it has a cube term and $\mathbf{A}$ lies in a congruence distributive variety. One can test whether $\mathbf{A}$ generates a congruence distributive variety in time polynomial in $|\mathbf{A}|$ for idempotent algebras (see [7] for the original algorithm or [1] for a more elementary algorithm) and in time exponential in $\mathbf{A}$ for general algebras (by taking the idempotent algorithm and adding the prefix seq $(A)$ to all tuples; see Lemma 14 below). As it happens, our cube term deciding algorithms also run in polynomial time (measured in $|\mathbf{A}|$ ) for an idempotent $\mathbf{A}$ and exponential time for a general $\mathbf{A}$ (see Theorem 23 and Corollary 37). We will return to near unanimity terms in Section 7.

To decide the existence of a cube term, we want to translate the problem from the language of operations into the language of relations. We have a good description of the shape of relations that, when compatible with an algebra $\mathbf{A}$, prevent $\mathbf{A}$ from having cube terms:

We say that an $n$-ary relation $R$ on $A$ is elusive if there exist tuples $\mathbf{a}, \mathbf{b} \in A^{n}$ such that $\chi_{I}(\mathbf{a}, \mathbf{b}) \in R$ for all $I \neq \emptyset$, but $\chi_{\emptyset}(\mathbf{a}, \mathbf{b})=\mathbf{a} \notin R$. In this situation, the tuple $\left(a_{1}, \ldots, a_{n}\right)$ is called an elusive tuple for $R$ (this is a notion similar to, but stricter than, essential tuples used in [17]; we note that elusive tuples were used, unnamed, already in [3]). Elusive relations prevent the existence of cube terms of low dimensions:

Observation 6. If $\mathbf{A}$ is an idempotent algebra which is compatible with an $n$-ary elusive relation, then $\mathbf{A}$ does not have any cube term of dimension $n$ or less.

Proof. Let $R \leq \mathbf{A}^{n}$ be elusive and let $\mathbf{a}, \mathbf{b}$ be a pair of tuples that witness the elusiveness of $R$.

Since we can trivially obtain an $n$ dimensional cube term from a cube term of lower dimension by introducing dummy variables, we only consider the case that $\mathbf{A}$ has a cube term of dimension $n$. Then applying this cube term on $\left(\chi_{I}(\mathbf{a}, \mathbf{b}): I \neq \emptyset\right)$ would give us $\mathbf{a} \in R$, a contradiction. 
Definition 7. Let $\mathbf{A}$ be an idempotent algebra. Then a pair $(C, D)$ is a cube term blocker (or just a "blocker" for short) if $C$ and $D$ are nonempty subuniverses of $\mathbf{A}, C \subsetneq D$ and $\forall n, D^{n} \backslash(D \backslash C)^{n} \leq \mathbf{A}^{n}$.

The reason for the name "cube term blockers" comes from the following result:

Proposition 8 ([14]). Let $\mathbf{A}$ be a finite idempotent algebra. Then $\mathbf{A}$ has a cube term if and only if it possesses no cube term blockers.

Viewed in $n$-dimensional space, the relation $D^{n} \backslash(D \backslash C)^{n}$ looks like a hypercube with one corner chipped off (the missing corner prevents cube terms from working properly). The following proposition gives a logically equivalent way to describe cube term blockers (note that the original paper 14] actually used this as the definition of cube term blockers and showed equivalence with our Definition (7).

Proposition 9 ([14, Lemma 3.2]). Let $\mathbf{A}=\left(A ; f_{1}, \ldots, f_{n}\right)$ be an idempotent algebra and let $\emptyset \neq C \subsetneq D$ be two subuniverses of $\mathbf{A}$. Denote the arity of $f_{i}$ by $m_{i}$. Then $(C, D)$ is a cube term blocker of $\mathbf{A}$ if and only if for each $i=1, \ldots, n$ there exists a coordinate $j$ between 1 and $m_{i}$ such that for each $c \in C$ and all $d_{1}, \ldots, d_{m_{i}} \in D$ we have

$$
f\left(d_{1}, \ldots, d_{j-1}, c, d_{j+1}, \ldots, d_{m_{i}}\right) \in C .
$$

Remark 10. Proposition 9 immediately gives us an algorithm (first described in [14) that decides in polynomial time if $(C, D)$ is a blocker for an algebra A given by its idempotent basic operations: First test if $\emptyset \neq C \subsetneq D$ and $C, D \leq \mathbf{A}$, then for every basic operation $f$ try out all the coordinates and see if $f(D, \ldots, D, C, D, \ldots, D) \subseteq C$ for some position of $C$. If we can find such a coordinate for all basic operations, then we have a blocker; else $(C, D)$ is not a blocker. The testing of coordinates can be implemented in time $O(m|\mathbf{A}|)$, by passing through each table of each operation exactly once and keeping track of the coordinates that each tuple rules out (here $m$ is the maximum arity of a basic operation of $\mathbf{A}$ ).

In the following, we will need a more general version of cube term blocker. Let $\emptyset \neq C_{i} \subsetneq D_{i} \subseteq A$ and $n_{i} \in \mathbb{N}$ where $i=1,2, \ldots, k$. We then define the $\left(n_{1}+\cdots+n_{k}\right)$-ary relation which we call a chipped cube by

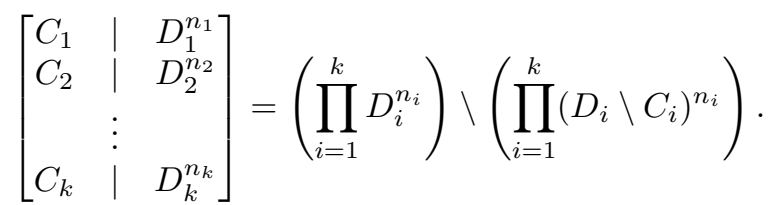

The coordinates of a chipped cube naturally break down into blocks: The $i$-th block, which we will often denote by $B_{i}$, consists of integers from $n_{1}+\cdots+$ $n_{i-1}+1$ to $n_{1}+\cdots+n_{i-1}+n_{i}$ (inclusively).

Remark 11. An important kind of relation employed in [12] is a "cross". It turns out that crosses are exactly those chipped cubes where the sets $D_{1}, \ldots, D_{k}$ are all equal to the whole universe of the algebra $\mathbf{A}$. 
We will sometimes omit unnecessary brackets as well as exponents equal to 1 , so for example we have

$$
\left[\begin{array}{c|c}
a & \{a, b\}^{2} \\
c & c, d
\end{array}\right]=\{a, b\}^{2} \times\{c, d\} \backslash\left\{\left(\begin{array}{l}
b \\
b \\
d
\end{array}\right)\right\} .
$$

The following two observations are immediate consequences of the definition of an elusive relation:

Observation 12. Let $\left(a_{1}, \ldots, a_{n}\right)$ be an elusive tuple for some relation $R$ compatible with the algebra $\mathbf{A}$. Then there exist elements $b_{1}, \ldots, b_{n}$ such that $\left(a_{1}, \ldots, a_{n}\right)$ is an elusive tuple for the relation

$$
S=\operatorname{Sg}\left(\left[\begin{array}{c}
b_{1} \mid a_{1}, b_{1} \\
b_{2} \mid a_{2}, b_{2} \\
\vdots \\
b_{n} \mid a_{n}, b_{n}
\end{array}\right]\right),
$$

and $S \subseteq R$.

Observation 13. Let $\mathbf{A}$ be an algebra. Let

$$
E=\left[\begin{array}{ccc}
C_{1} & \mid & D_{1}^{n_{1}} \\
C_{2} & \mid & D_{2}^{n_{2}} \\
& \vdots & \\
C_{k} & \mid & D_{k}^{n_{k}}
\end{array}\right]
$$

be a chipped cube where each $D_{1}, \ldots, D_{k}$ is a subuniverse of $\mathbf{A}$ and assume that the relation $\operatorname{Sg}(E)$ is not elusive. Then

$$
\operatorname{Sg}(E)=D_{1}^{n_{1}} \times D_{2}^{n_{2}} \times \cdots \times D_{k}^{n_{k}} .
$$

\section{General cube term results}

The following lemma is a variant of several previously known versions of the local-to-global arguments for idempotent algebras, see [14, Theorem 2.8] and 9]; the first condition is the global and the second the local one. The "prefix" $\operatorname{seq}(A)$ serves here to let us argue about general algebras as if they were idempotent.

The key observation to use the previous local-to-global results for idempotent algebras is to note that the tuples of $\operatorname{Sg}\left(\left\{\mathbf{u}: \mathbf{u}=\operatorname{seq}(A) \chi_{I}(\mathbf{a}, \mathbf{b}), I \neq\right.\right.$ $\emptyset\})$ that begin with $\mathbf{s e q}(A)$ are generated using the idempotent reduct of $\mathbf{A}$.

Lemma 14. Let $\mathbf{A}$ be a finite algebra. Then the following are equivalent:

(1) A has a cube term of dimension d,

(2) For all $\mathbf{a}, \mathbf{b} \in A^{d}$ we have

$$
\operatorname{seq}(A) \mathbf{a} \in \operatorname{Sg}\left(\left\{\mathbf{u}: \mathbf{u}=\operatorname{seq}(A) \chi_{I}(\mathbf{a}, \mathbf{b}), I \neq \emptyset\right\}\right) .
$$


Proof. By applying a cube term on the subalgebra in 2 it is immediate to see that (11) implies (2).

For the other implication, we essentially just cite [9]. Let $\mathbf{A}_{i d}$ be the idempotent reduct of $\mathbf{A}$ - the algebra on $A$ whose basic operations are all the idempotent operations from the clone of $\mathbf{A}$. It is easy to see that the condition (2) is equivalent to

(3) For all $\mathbf{a}, \mathbf{b} \in A^{d}$ we have $\mathbf{a} \in \operatorname{Sg}_{\mathbf{A}_{i d}}\left(\left\{\chi_{I}(\mathbf{a}, \mathbf{b}): I \neq \emptyset\right\}\right)$.

Let $E$ be the $d \times\left(2^{d}-1\right)$ matrix with columns indexed by nonempty subsets of $[n]$ whose $I$-th column is $\chi_{I}(y, x)$ for variable symbols $x, y$. Then the condition (3) on $\mathbf{A}_{i d}$ is what [9, Definition 2.2] calls having all local $E$ special cube terms on sets of size $d$. It is easy to verify that our $E$ satisfies the DCP [9, Definition 2.5] and hence using [9, Theorem 2.6] the matrix E has the local-to-global property of size $d$ [9, Definition 2.3] and hence if $\mathbf{A}_{i d}$ satisfies (3) (or 2) then $\mathbf{A}_{i d}$ has a cube term. Since the clone of operations of $\mathbf{A}_{i d}$ is a subset of the clone of operations of $\mathbf{A}$, it follows that $\mathbf{A}$ also has a cube term.

The following lemma sheds some light on what minimal compatible elusive relations look like. The additional assumption that we are dealing with a chipped cube will be justified later in Lemma 16.

Lemma 15. Let $\mathbf{A}$ be an algebra, $R \subseteq A^{n}$ be inclusion minimal among elusive relations compatible with $\mathbf{A}$ (that is, no proper subset of $R$ is both an elusive relation and compatible with $\mathbf{A})$. Assume moreover that $R$ is equal to the chipped cube

$$
\left[\begin{array}{ccc}
C_{1} & \mid & D_{1} \\
& \vdots & \\
C_{n} & \mid & D_{n}
\end{array}\right] .
$$

Let $\mathbf{a}=\left(a_{1}, \ldots, a_{n}\right)$ and $\mathbf{b}=\left(b_{1}, \ldots, b_{n}\right)$ be two tuples witnessing the elusiveness of $R$. Then:

(a) $R=\operatorname{Sg}\left(\left\{\chi_{I}(\mathbf{a}, \mathbf{b}): \emptyset \neq I \subseteq[n]\right\}\right)$,

(b) there is no subuniverse $E$ of $\mathbf{A}$ such that $C_{i} \subsetneq E \subsetneq D_{i}$ for some $i$; in particular $D_{i}=\operatorname{Sg}\left(a_{i}, b_{i}\right)$ for each $i$,

(c) if $i, j$ are such that $\left(a_{i}, b_{i}\right)=\left(a_{j}, b_{j}\right)$, then $\left(C_{i}, D_{i}\right)=\left(C_{j}, D_{j}\right)$,

(d) if $D_{i}=D_{j}$ for some $i$ and $j$ then either $C_{i}=C_{j}$ or $C_{i} \cap C_{j}=\emptyset$.

Proof. Note that for each $i$ we have $a_{i} \in D_{i} \backslash C_{i}$ and $b_{i} \in C_{i}$.

Part (国) follows from the fact that $\operatorname{Sg}\left(\left\{\chi_{I}(\mathbf{a}, \mathbf{b}): \emptyset \neq I \subseteq[n]\right\}\right)$ is the smallest compatible relation that contains all the tuples that witness the elusiveness of $R$.

The proof of point (b) is similar. Were there $E$ strictly between $C_{i}$ and $D_{i}$, we could restrict the $i$-th coordinate of $R$ to $E$ and obtain a smaller elusive compatible relation, proving (b).

To see that (드) holds, take $i$ and $j$ such that $a_{i}=a_{j}$ and $b_{i}=b_{j}$. Without loss of generality let $i<j$. Then the set of generators of $R$ is invariant 
under the permutation that swaps $i$-th and $j$-th coordinates. Therefore, $R$ is invariant under such a permutation of coordinates as well. Consider now

$$
R^{\prime}=\left\{(e, f):\left(a_{1}, \ldots, a_{i-1}, e, a_{i+1}, \ldots, a_{j-1}, f, a_{j+1}, \ldots, a_{n}\right) \in R\right\} .
$$

Since $R$ is a chipped cube, it follows that $R^{\prime}=C_{i} \times D_{j} \cup D_{i} \times C_{j}$ and from the symmetry of $R$, we get that $R^{\prime}$ is symmetric as a binary relation. It is straightforward to verify that this can only happen when $C_{i}=C_{j}$ and $D_{i}=D_{j}$.

To prove part (d), assume (without loss of generality) that $D_{1}=D_{2}=$ $D$ and there exist $e \in C_{1} \backslash C_{2}$ and $c \in C_{1} \cap C_{2}$.

Consider then the chipped cube we get from $R$ by switching the first two coordinates:

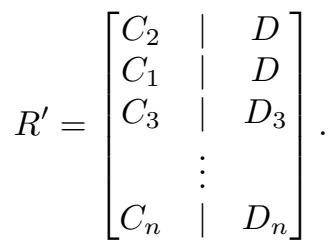

By symmetry, $R^{\prime}$ is a relation compatible with $\mathbf{A}$. This makes $R \cap R^{\prime}$ also compatible with $\mathbf{A}$. We now claim that $R \cap R^{\prime}$ is an elusive relation that is strictly smaller than $R$. To see $R \cap R^{\prime} \subsetneq R$, choose $d_{i} \in D_{i} \backslash C_{i}$ for $i=2, \ldots, n$ and observe that $R$ contains the tuple $e d_{2} d_{3} \cdots d_{n}$, while $R \cap R^{\prime}$ does not. To prove that $R \cap R^{\prime}$ is elusive, choose $c_{i} \in C_{i}$ for $i=3,4, \ldots, n$ and observe that $R \cap R^{\prime}$ contains the tuple $\chi_{I}\left(e d_{2} d_{3} \cdots d_{n}, c c c_{3} \cdots c_{n}\right)$ for any $I \neq \emptyset$, but not for $I=\emptyset$. This concludes the proof of the last point.

In the proof above we made progress thanks to swapping two coordinates of $R$. Later in the paper, we will be working with a general mapping that moves coordinates of tuples around.

\section{Cube terms in idempotent algebras}

Lemma 16. Let $\mathbf{A}$ be an idempotent algebra, $R$ a relation that is inclusion minimal among elusive relations compatible with $\mathbf{A}$. Then $R$ is a chipped cube.

Proof. Given that $R$ is minimal, our strategy will be to fit a maximal chipped cube into $R$ and show that this chipped cube is equal to $R$.

Let $\left(a_{1}, \ldots, a_{n}\right)$ be an elusive tuple for $R$. Let moreover

$$
E=\left[\begin{array}{ccc}
C_{1} & \mid & D_{1} \\
& \vdots & \\
C_{n} & \mid & D_{n}
\end{array}\right]
$$

be an inclusion-maximal chipped cube such that (1) $E \subseteq R$ and (2) $a_{i} \in D_{i}$ for each $i=1,2, \ldots, n$. (At least one such chipped cube exists by Observation 12.) 
We prepare ground for our proof by exploring properties of the sets $D_{i}$. From the maximality of $E$, it follows that each of the sets $D_{i}$ is a subuniverse of A: Were, say, $u \in \operatorname{Sg}\left(D_{1}\right) \backslash D_{1}$ then $u=t\left(d_{1}, \ldots, d_{m}\right)$ for some operation $t$ of $\mathbf{A}$ and suitable $d_{1}, \ldots, d_{m} \in D_{1}$. Take any $e_{2}, \ldots, e_{n} \in A$ such that for each $i$ we have $e_{i} \in D_{i}$ and for at least one $i \in\{2, \ldots, n\}$ we have $e_{i} \in C_{i}$. By definition of a chipped cube, we have $\left(d_{j}, e_{2}, \ldots, e_{n}\right) \in E$ for each $j=1, \ldots, m$; applying $t$ thus gives us $\left(u, e_{2}, \ldots, e_{n}\right) \in \operatorname{Sg}(E)$. Therefore $\operatorname{Sg}(E) \subseteq R$ contains the chipped cube

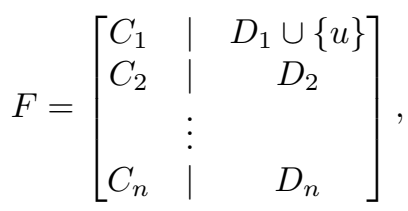

a contradiction with the maximality of $E$.

From the minimality of $R$, we immediately get $R=\operatorname{Sg}(E)$ and what is more $\pi_{i}(R)=\operatorname{Sg}\left(C_{i} \cup\left\{a_{i}\right\}\right)$ for each $i$. The latter equality follows from the fact that $R \cap \prod_{i=1}^{n} \operatorname{Sg}\left(C_{i} \cup\left\{a_{i}\right\}\right)$ is an elusive relation compatible with $\mathbf{A}$ and we chose $R$ to be a minimal elusive relation compatible with $\mathbf{A}$. Since for each $i$ we have $\operatorname{Sg}\left(C_{i} \cup\left\{a_{i}\right\}\right) \subseteq D_{i} \subseteq \pi_{i}(R)$ and the outer pair of sets is equal, it follows that actually $D_{i}=\operatorname{Sg}\left(C_{i} \cup\left\{a_{i}\right\}\right)=\pi_{i}(R)$ for each $i$.

We are now ready to show that $E=R$. Assume otherwise and choose $\mathbf{u} \in R \backslash E$ that agrees with $\left(a_{1}, a_{2}, \ldots, a_{n}\right)$ on as many coordinates as possible. Up to reordering of coordinates, we thus have a tuple $\mathbf{u}=\left(u_{1}, u_{2}, \ldots, u_{n}\right) \in$ $R \backslash E$ such that $\left(a_{1}, u_{2}, \ldots, u_{n}\right) \notin R$.

Since $\mathbf{u} \in R \backslash E$, we see that $u_{i} \in D_{i} \backslash C_{i}$ for all $i=1, \ldots, n$. We will show that $R$ contains the chipped cube

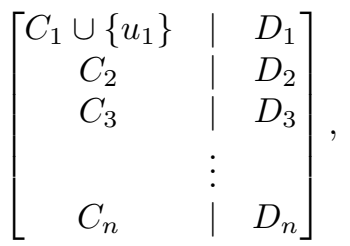

yielding a contradiction with the maximality of $E$. To prove this, we need to show that $\left\{u_{1}\right\} \times D_{2} \times \cdots \times D_{n} \subseteq R$.

Observe first that $\operatorname{Sg}\left(C_{1} \cup\left\{u_{1}\right\}\right) \times\left\{\left(u_{2}, \ldots, u_{n}\right)\right\} \subseteq R$. This follows from the inclusion $\left(C_{1} \cup\left\{u_{1}\right\}\right) \times\left\{\left(u_{2}, \ldots, u_{n}\right)\right\} \subseteq R$ and the idempotence of $\mathbf{A}$.

Let $D^{\prime}=\operatorname{Sg}\left(C_{1} \cup\left\{u_{1}\right\}\right)$. Since $\left(a_{1}, u_{2}, \ldots, u_{n}\right) \notin R$, we obtain $a_{1} \notin D^{\prime}$, making $D^{\prime}$ strictly smaller than $D_{1}$. 
Consider now the relation $R^{\prime}=R \cap\left(D^{\prime} \times D_{2} \times D_{3} \times \cdots \times D_{n}\right)$. From $R^{\prime} \subsetneq R$ we see that $R^{\prime}$ is not elusive. However, $R^{\prime}$ contains the chipped cube

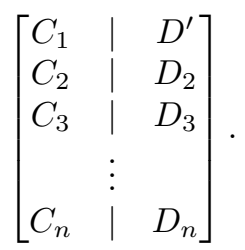

This is only possible if $\left\{u_{1}\right\} \times D_{2} \times \cdots \times D_{n} \subseteq R^{\prime} \subseteq R$, which is exactly what we needed.

We would like to remark that Lemma [16] does not hold for general algebras. As an example, consider the algebra $\mathbf{A}$ on the set $\{0,1,2\}$ with one unary constant operation $c_{2}(x)=2$. For any $n \geq 2$ let

$$
R=\left\{2^{\mathbf{n}}\right\} \cup\{0,1\}^{n} \backslash\left\{10^{\mathbf{n}-\mathbf{1}}\right\} .
$$

This relation is compatible with $\mathbf{A}$ and elusive. It is straightforward to verify that $R$ is also minimal such $(n$-ary elusive relation needs to contain at least $2^{n}-1$ tuples; $R$ is just one tuple larger than this theoretical minimum, and a case consideration shows that we can't discard any tuple from $R$ ). However, $R$ is not a chipped cube: The projections of $R$ to each coordinate are all equal to $\{0,1,2\}$ and $R$ contains the tuple $2^{\mathbf{n}}$. It is not hard to show that any chipped cube with these two properties contains at least $3^{n-1}$ tuples with at least one entry 2 , a property that $R$ fails to have.

Corollary 17. Let $\mathbf{A}$ be a finite idempotent algebra, and let $d \geq 2$ be an integer. Then $\mathbf{A}$ has a cube term of dimension $d$ if and only if there is no $d$-ary chipped cube relation compatible with $\mathbf{A}$.

Proof. Cube terms are incompatible with chipped cubes, so the interesting implication is that the absence of a $d$-dimensional cube term gives us a $d$-ary compatible chipped cube.

Assume thus that $\mathbf{A}$ has no $d$-dimensional cube term. We claim that then $\mathbf{A}$ is compatible with some $d$-ary elusive relation. By Lemma 14, there exist $\mathbf{a}, \mathbf{b} \in A^{d}$ such that $\operatorname{seq}(A) \mathbf{a} \notin \operatorname{Sg}\left(\left\{\operatorname{seq}(A) \chi_{I}(\mathbf{a}, \mathbf{b}): I \neq \emptyset\right\}\right)$. Since $\mathbf{A}$ is idempotent, we can remove the $\mathbf{s e q}(A)$ prefix and have $\mathbf{a} \notin \operatorname{Sg}\left(\left\{\chi_{I}(\mathbf{a}, \mathbf{b}): I \neq\right.\right.$ $\emptyset\})$. This amounts to saying that $\mathbf{a}$ is an elusive tuple for $\operatorname{Sg}\left(\left\{\chi_{I}(\mathbf{a}, \mathbf{b}): I \neq\right.\right.$ $\emptyset\})$. To finish the proof, we take an inclusion minimal $d$-ary elusive relation $E$ compatible with A. By Lemma 16, this $E$ is a chipped cube.

The following Lemma is a nontrivial consequence of Proposition 9 .

Lemma 18. Let $\mathbf{A}=\left(A ; f_{1}, \ldots, f_{\ell}\right)$ be an idempotent algebra that has a cube term. Denote by $m_{1}, \ldots, m_{\ell}$ the arities of $f_{1}, \ldots, f_{\ell}$. Assume that a chipped 
cube

is compatible with $\mathbf{A}$. Let

$$
F=\left[\begin{array}{ccc}
C_{1} & \mid & D_{1} \\
C_{2} & \mid & D_{2} \\
& \vdots & \\
C_{k} & \mid & D_{k}
\end{array}\right]
$$

$U_{i}=\left\{j:\left(C_{j}, D_{j}\right)\right.$ is not a cube term blocker in the algebra $\left.\left(A ; f_{i}\right)\right\}$

where $i$ goes from 1 to $\ell$ (see Definition 7 for what a cube term blocker is). Then:

(a) $\bigcup_{i=1}^{\ell} U_{i}=[k]$, and

(b) for each $i=1, \ldots$, $\ell$ we have $m_{i} \geq 1+\left|U_{i}\right|$.

Proof. Note that we implicitly have $\emptyset \neq C_{i} \subsetneq D_{i}$ for each $i$ from the definition of a chipped cube.

Since $\mathbf{A}$ has a cube term, there is no cube term blocker in $\mathbf{A}$. In particular none of $\left(C_{1}, D_{1}\right),\left(C_{2}, D_{2}\right), \ldots,\left(C_{k}, D_{k}\right)$ are blockers for $\left(A ; f_{1}, \ldots, f_{\ell}\right)$. Were, say, $\left(C_{1}, D_{1}\right)$ a blocker for all algebras $\left(A ; f_{i}\right)$ then each $f_{i}$ maps each relation $D_{1}^{n} \backslash\left(D_{1} \backslash C_{1}\right)^{n}$ into itself, making $\left(C_{1}, D_{1}\right)$ a blocker for the whole algebra $\mathbf{A}$. Therefore, for each $j \in[k]$ there exists an $i$ so that $j \in U_{i}$, i.e. $\bigcup_{i=1}^{\ell} U_{i}=[k]$, giving us part (国).

It remains to show that for each $i \in[\ell]$ we have $m_{i} \geq 1+\left|U_{i}\right|$. Assume for a contradiction that $m_{i} \leq\left|U_{i}\right|$ for some $i$. Without loss of generality we can assume that in fact $\left[m_{1}\right] \subseteq U_{1}$ (we are free to reorder the $f_{i}$ 's and $\left(C_{j}, D_{j}\right)$ 's). We then consider the chipped cube

$$
E=\left[\begin{array}{ccc}
C_{1} & \mid & D_{1} \\
C_{2} & \mid & D_{2} \\
& \vdots & \\
C_{m_{1}} & \mid & D_{m_{1}}
\end{array}\right] .
$$

It is easy to see that $E$ can be obtained from $F$ by restricting all but the first $m_{1}$ coordinates to some singleton values from $D_{j} \backslash C_{j}$ and projecting the result to the first $m_{1}$ coordinates (here we need that $\mathbf{A}$ is idempotent). Therefore $f_{1}$ preserves $E$.

However, we know that $\left(C_{1}, D_{1}\right), \ldots,\left(C_{m_{1}}, D_{m_{1}}\right)$ are not blockers for $\left(A ; f_{1}\right)$. By Proposition [9, for each $j \in\left\{1,2, \ldots, m_{1}\right\}$ we then can find a tuple

$$
\left(a_{j, 1}, a_{j, 2}, \ldots, a_{j, m_{1}}\right) \in D_{j}^{m_{1}}
$$

such that $a_{j, j} \in C_{j}$ and $f_{1}\left(a_{j, 1}, \ldots, a_{j, m_{1}}\right) \notin C_{j}$.

Arrange the above mentioned tuples into rows of an $m_{1} \times m_{1}$ matrix $M$. Since $a_{j, j} \in C_{j}$ for all $j=1, \ldots, m_{1}$, each column of $M$ belongs to $E$. Therefore, we should have $f_{1}(M) \in E$ as well. But $f_{1}$ applied to the $j$-th row of the matrix $M$ gives us an element from $D_{j} \backslash C_{j}$ for each $j$, so $f_{1}(M)$ fails to be in $E$, a contradiction. 
Since the sets $U_{i}$ in the above theorem depend only on $f_{i}$ and the sets $C_{j}, D_{j}$, we can generalize the result to the case when $\left(C_{j}, D_{j}\right)$ appears multiple times in $F$ :

Corollary 19. Let $\mathbf{A}=\left(A ; f_{1}, \ldots, f_{\ell}\right)$ be an idempotent algebra that has a cube term. Denote by $m_{1}, \ldots, m_{\ell}$ the arities of $f_{1}, \ldots, f_{\ell}$. Assume that a chipped cube

$$
F=\left[\begin{array}{ccc}
C_{1} & \mid & D_{1}^{n_{1}} \\
C_{2} & \mid & D_{2}^{n_{2}} \\
& \vdots & \\
C_{k} & \mid & D_{k}^{n_{k}}
\end{array}\right]
$$

is compatible with $\mathbf{A}$. Then there exists a family of sets $U_{1}, \ldots, U_{\ell}$ such that $\bigcup_{i=1}^{\ell} U_{i}=[k]$ and for each $i$ we have $m_{i} \geq 1+\sum_{j \in U_{i}} n_{j}$.

We are now ready to give a lower bound on the dimension of a cube term in finite idempotent algebras with cube terms. For a version of the following theorem that works for infinite idempotent algebras, see [12, Theorem 4.1] (we discuss the relationship between our paper and [12 in detail at the end of the Introduction).

Theorem 20. Let $\mathbf{A}=\left(A ; f_{1}, \ldots, f_{\ell}\right)$ be a finite idempotent algebra. Let $m_{1} \geq$ $m_{2} \geq \cdots \geq m_{\ell}$ be the arities of the basic operations of $\mathbf{A}$. Let $N=1+$ $\sum_{i=1}^{r}\left(m_{i}-1\right)$ where $r=\min \left(\ell,\left(\begin{array}{c}|A| \\ 2\end{array}\right)\right)$. Assume that $N>2$ or $|A|>2$. Then the following are equivalent:

(a) A does not have a cube term.

(b) A has a cube term blocker.

(c) A does not have a cube term of dimension $N$.

(d) There is an $N$-ary elusive relation compatible with $\mathbf{A}$.

(e) There exists an $N$-ary chipped cube compatible with $\mathbf{A}$.

Proof. The equivalence of (a) and (b) is already known [3].

If (b) holds and $(C, D)$ is a cube term blocker for $\mathbf{A}$, then the relation $D^{N} \backslash(D \backslash C)^{N}$ is an $N$-ary chipped cube, proving ([e]).

From (E), condition (dd) follows trivially, from which (c) follows imme-

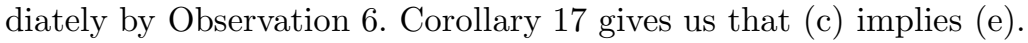

It remains to show that (远) implies (a). We proceed by contradiction, assuming that $\mathbf{A}$ has both a cube term and an $N$-ary compatible chipped cube. Take $n$ smallest such that $\mathbf{A}$ has a cube term of dimension $n+1$. Certainly $n \geq N$ since chipped cubes are elusive relations.

Since $\mathbf{A}$ is idempotent without an $n$-dimensional cube term, there must exist an $n$-ary elusive relation $R$ compatible with $\mathbf{A}$. Let $\mathbf{a}, \mathbf{b}$ be the tuples that witness the elusiveness of $R$. We choose $R$ to be inclusion minimal; by Lemma 16 $R$ is a chipped cube. Applying Lemma 15, we get that

$$
E=\left[\begin{array}{lll}
C_{1} & \mid & D_{1} \\
& \vdots & \\
C_{n} & \mid & D_{n}
\end{array}\right],
$$


where $D_{i}=\operatorname{Sg}\left(a_{i}, b_{i}\right)$ for all $i \in[n]$ and $\left(C_{i}, D_{i}\right)=\left(C_{j}, D_{j}\right)$ whenever $\left(a_{i}, b_{i}\right)=\left(a_{j}, b_{j}\right)$. Let us reorder the coordinates of $\mathbf{a}, \mathbf{b}$ so that identical pairs $\left(a_{i}, b_{i}\right)$ are grouped together. Let $k$ be the number of distinct pairs $\left(a_{i}, b_{i}\right)$ and denote by $n_{i}$ the number of times the $i$-th pair appears. After this reordering (and renaming of $C_{i}, D_{i}$ 's) we have

$$
E=\left[\begin{array}{ccc}
C_{1} & \mid & D_{1}^{n_{1}} \\
& \vdots & \\
C_{k} & \mid & D_{k}^{n_{k}}
\end{array}\right],
$$

where of course $n_{1}+n_{2}+\cdots+n_{k}=n$. Since $a_{i} \neq b_{i}$ for each $i$, we obtain $k \leq|A|(|A|-1)$.

Assume first that $N>2$ (we will deal with the special cases $N=2$ and $N=1$ later). We claim that then in fact $k \leq|A|(|A|-1) / 2$. To prove this, we show that for all $i, j$ we have $\left(a_{i}, b_{i}\right) \neq\left(b_{j}, a_{j}\right)$. For $i=j$, this is obvious, so assume without loss of generality that $i=1, j=n$. Then $D_{1}=D_{n}=\operatorname{Sg}\left(a_{1}, a_{n}\right)$ and $C_{1} \cap C_{n}=\emptyset$ by Lemma 15. Consider the relation

$$
F=\left\{\left(x_{1}, \ldots, x_{2 n-2}\right): \exists z \in D_{1},\left(x_{1}, \ldots, x_{n-1}, z\right),\left(z, x_{n}, \ldots, x_{2 n-2}\right) \in E\right\} .
$$

From the definition of $F$ we see that it is compatible with $\mathbf{A}$. We claim that the tuple $\left(a_{1}, a_{2}, \ldots, a_{n-1}, a_{2}, a_{3}, \ldots, a_{n}\right)$ is elusive for $F$. This tuple is not a member of $F$ because were $\left(a_{1}, \ldots, a_{n-1}, a_{2}, \ldots, a_{n}\right) \in F$, then there would exist a $z$ such that $\left(a_{1}, \ldots, a_{n-1}, z\right),\left(z, a_{2}, \ldots, a_{n}\right) \in E$. However, such $z$ would need to lie in both $C_{1}$ and $C_{n}$, a contradiction. On the other hand, if we rewrite one or more entries of $\left(a_{1}, \ldots, a_{n-1}, a_{2}, \ldots, a_{n}\right)$ to $b_{i}$, we can choose $z=b_{n}$ or $z=b_{1}$ and satisfy both $\left(x_{1}, \ldots, x_{n-1}, z\right) \in E$ and $\left(z, x_{2}, \ldots, x_{n}\right) \in$ E.

Since $n \geq N>2$, we have $2 n-2>n$, and so $F$ is an elusive compatible relation of arity higher than $n$, a contradiction with $\mathbf{A}$ having an $(n+1)$ dimensional cube term. Therefore $k \leq|A|(|A|-1) / 2$.

We now apply Corollary 19 to the relation

$$
E=\left[\begin{array}{ccc}
C_{1} & \mid & D_{1}^{n_{1}} \\
& \vdots & \\
C_{k} & \mid & D_{k}^{n_{k}}
\end{array}\right],
$$

obtaining a family of sets $U_{1}, \ldots, U_{\ell}$ (one for each basic operation of $\mathbf{A}$ ) such that $U_{1}, \ldots, U_{\ell}$ cover $[k]$ and $m_{i}-1 \geq \sum_{j \in U_{i}} n_{j}$ for each $i$. Take $r=$ $\min \left(\ell,\left(\begin{array}{c}|A| \\ 2\end{array}\right)\right)$ and observe that we can then cover $[k]$ by at most $r$ sets chosen from $U_{1}, \ldots, U_{\ell}$. (If $r=\left(\begin{array}{c}|A| \\ 2\end{array}\right)<\ell$, the existence of such a covering of $[k]$ follows from $k \leq\left(\begin{array}{c}|A| \\ 2\end{array}\right)$.) Let $I \subseteq[\ell]$ be a set of indices of size $r$ so that $\bigcup_{i \in I} U_{i}=[k]$. Since the arities $m_{1}, \ldots, m_{\ell}$ are at least one and ordered in a nonincreasing order, we get the inequalities:

$$
\sum_{i=1}^{r}\left(m_{i}-1\right) \geq \sum_{i \in I}\left(m_{i}-1\right) \geq \sum_{j=1}^{k} n_{j}=n .
$$


Therefore, $N-1=\sum_{i=1}^{r}\left(m_{i}-1\right) \geq n$, a contradiction with the assumption $n+1>N$.

Assume now that $N=2$. Given the formula for $N$, we must have $m_{1}=2$ and $m_{2}=\cdots=m_{\ell}=1$. (Note that we are using $|A|>2$ here; we need $r \geq 2$ to make sure that $m_{2}=1$.) Since $\mathbf{A}$ is idempotent, the operations $f_{2}, \ldots, f_{\ell}$ are unary identity mappings - without loss of generality let $\ell=1$. We again take $n \geq N=2$ such that $n+1$ is the least dimension of a cube term in $\mathbf{A}$ and construct the chipped cube $E$ as above. We only have the $k \leq|A|(|A|-1)$ bound in this case; however, we can finish the proof anyway: We apply Corollary 19 with $\ell=1$ and $m_{1}=2$ to get that $2=m_{1} \geq$ $1+n_{1}+\cdots+n_{k}=1+n$, i.e. $1 \geq n$. This is a contradiction with $n \geq N=2$, finishing the proof.

Finally, if $N=1$, the clone of operations of $\mathbf{A}$ consists of projections only and both (迆) and (国) are trivially true.

Using Theorem 20, we can obtain a bound on the dimension of a cube term that depends quadratically on $|A|$ and linearly on the maximum arity of a basic operation of $\mathbf{A}$.

Corollary 21. Let A be finite idempotent algebra whose basic operations have a maximal arity $m$. Then $\mathbf{A}$ has a cube term if and only if $\mathbf{A}$ has a cube term of dimension at most $1+(m-1)\left(\begin{array}{c}|A| \\ 2\end{array}\right)$.

Proof. By Theorem 20, if $\mathbf{A}$ has a cube term blocker, it has a cube term blocker of arity at most $N=1+\sum_{i=1}^{r}\left(m_{i}-1\right)$ where each $m_{i} \leq m$ and $r \leq$ $\left(\begin{array}{c}|A| \\ 2\end{array}\right)$. Hence, we can estimate the arity of the cube term as $N \leq 1+(m-1)\left(\begin{array}{c}|A| \\ 2\end{array}\right)$ and the claim follows.

It turns out that the bound on the dimension of a cube term in Theorem 20 and Corollary 21] is tight (see also [12, Example 4.4]):

Theorem 22. Let $\ell, n \in \mathbb{N}$ and $m_{1} \geq m_{2} \geq \cdots \geq m_{\ell}$ be positive integers. Let $N=1+\sum_{i=1}^{r}\left(m_{i}-1\right)$ where $r=\min \left(\ell,\left(\begin{array}{l}n \\ 2\end{array}\right)\right)$. Assume that either $n>2$ or $n=2$ and $N>2$. Then there exist idempotent operations $f_{1}, \ldots, f_{\ell}$ on the set $[n]$ of arities $m_{1}, \ldots, m_{\ell}$ such that $\mathbf{A}=\left([n] ; f_{1}, \ldots, f_{\ell}\right)$ has a cube term of dimension $N$, but no cube term of dimension $N-1$.

Proof. Without loss of generality assume that all $m_{i}$ are at least 2 (unary idempotent operations are equal to the identity mapping and therefore not interesting).

Let us handle the case $n>2$ and $N=2$ first. Since there is no cube term of dimension $N-1=1$, all we need to do is produce an algebra on $n$ elements with one basic idempotent operation of arity 2 and a Maltsev term (i.e. a cube term of dimension 2). We choose $\mathbf{A}=([n], f)$ to be an idempotent quasigroup of order $n$. Such a quasigroup exists for all $n>2$ (see [13, Theorem 2.2.3]) and all quasigroups have a Maltsev operation.

Assume now that $N>2$ and $n \geq 2$. Partition the set $\left\{(a, b) \in[n]^{2}: a<\right.$ $b$ into $r$ (nonempty, disjoint) sets $J_{1}, \ldots, J_{r}$ (such a partition will exist because $\left.r \leq\left(\begin{array}{c}n \\ 2\end{array}\right)\right)$. We then define the operations $f_{1}, \ldots, f_{r}$ as follows: For each 
$i=1,2, \ldots, r$, let

$$
f_{i}(a, a, \ldots, a, b)=\cdots=f_{i}(b, a, \ldots, a, a)=a
$$

for all pairs $(a, b) \in J_{i}$. Otherwise, let $f_{i}\left(x_{1}, \ldots, x_{m_{1}}\right)=\max \left(x_{1}, \ldots, x_{m_{1}}\right)$. If $\ell>r$, we choose the remaining operations $f_{r+1}, \ldots, f_{\ell}$ to be projections to the first coordinate.

We now claim that the algebra $\mathbf{A}=\left([n] ; f_{1}, \ldots, f_{\ell}\right)$ has an $N$-dimensional cube term, but no $(N-1)$-dimensional cube term.

To prove that $\mathbf{A}$ has an $N$-dimensional cube term, it is enough to show that there is no cube term blocker in $\mathbf{A}$ and apply Theorem 20 Let $\emptyset \neq C \subsetneq$ $D \subseteq A$ be a candidate for a blocker. Pick a pair $c \in C, d \in D \backslash C$. We will show that there is an $i$ such that

$$
f_{i}(c, d, \ldots, d, d)=f_{i}(d, c, \ldots, d, d)=\cdots=f_{i}(d, d, \ldots, d, c)=d \notin C,
$$

which contradicts Proposition 9 .

If $c<d$, we do the following. If the map $f_{1}$ is at least ternary then $f_{1}(c, d, \ldots, d, d)=f_{1}(d, c, \ldots, d, d)=\cdots=f_{1}(d, d, \ldots, d, c)=\max (c, d)=d$. If $m_{1}=2$, then $m_{2}=2$ (because of $N>2$ ) and for some $j \in\{1,2\}$ we have $f_{j}(c, d)=f_{j}(d, c)=\max (c, d)=d$.

If $c>d$, then there exists an $i$ such that $(d, c) \in J_{i}$ and so from the definition of $f_{i}$ 's we have

$$
f_{i}(c, d, \ldots, d, d)=f_{i}(d, c, \ldots, d, d)=\cdots=f_{i}(d, d, \ldots, d, c)=d .
$$

It remains to show that $\mathbf{A}$ has no cube term of dimension $N-1$. We do this by constructing an $(N-1)$-ary elusive relation that is compatible with A. Pick a pair $\left(a_{i}, b_{i}\right) \in J_{i}$ for every $i=1,2, \ldots, r$ and consider the $N$-tuples $\mathbf{a}=a_{1}^{\mathbf{m}_{\mathbf{1}}-\mathbf{1}} a_{2}^{\mathbf{m}_{\mathbf{2}}-\mathbf{1}} \ldots a_{r}^{\mathbf{m}_{\mathbf{r}}-\mathbf{1}}$ and $\mathbf{b}=b_{1}^{\mathbf{m}_{\mathbf{1}}-\mathbf{1}} b_{2}^{\mathbf{m}_{\mathbf{2}}-\mathbf{1}} \ldots b_{r}^{\mathbf{m}_{\mathbf{r}}-\mathbf{1}}$. We claim that all operations $f_{1}, \ldots, f_{r}$ map $R=\left\{\chi_{I}(\mathbf{a}, \mathbf{b}): I \neq \emptyset\right\}$ into itself. This will conclude the proof, since $R$ is elusive (as witnessed by $\mathbf{a} \notin R$ ).

We will show that $f_{1}$ maps $R$ into itself; the proof for $f_{2}, \ldots, f_{r}$ is similar. Since $f_{1}$ is conservative (it always returns one of its arguments) we only have to show that there is no $(N-1) \times m_{1}$ matrix $M$ with columns from $R$ such that $f_{1}(M)=\mathbf{a}$. Since the first $m_{1}-1$ entries of a are $a_{1}$ and $f_{1}$ only returns $a_{1}$ when at most one of inputs differs from $a_{1}$, there can be at most $m_{1}-1$ entries different from $a_{1}$ in the top $\left(m_{1}-1\right) \times m_{1}$ submatrix of $M$. Since $M$ has $m_{1}$ columns there is a column $\mathbf{c}$ of $M$ that begins with $a_{1}^{\mathbf{m}_{1}}$. Let us examine this column $\mathbf{c}$ more closely.

Since $\mathbf{c} \in R$, there has to be $i>1$ such that the $i$-th block of $\mathbf{c}$ contains $b_{i}$. Since $b_{i}>a_{i}$ and $\left(a_{i}, b_{i}\right) \notin J_{1}$ (this follows from $\left.J_{i} \cap J_{1} \neq \emptyset\right), f_{1}$ applied to $b_{i}$ and any combination of $a_{i}$ 's and $b_{i}$ 's will return $b_{i}$. Therefore, the $i$-th block of $f_{1}(M)$ also contains $b_{i}$, yielding $f_{1}(M) \neq \mathbf{a}$ as we needed.

What happens to the claims of Theorem 20 when $|A|, N \leq 2$ ? If $N=$ 1 or $|A|=1$ the algebra $\mathbf{A}$ is not very interesting since it contains only projections. When $|A|=N=2$, we can have algebras that have a cube term, 


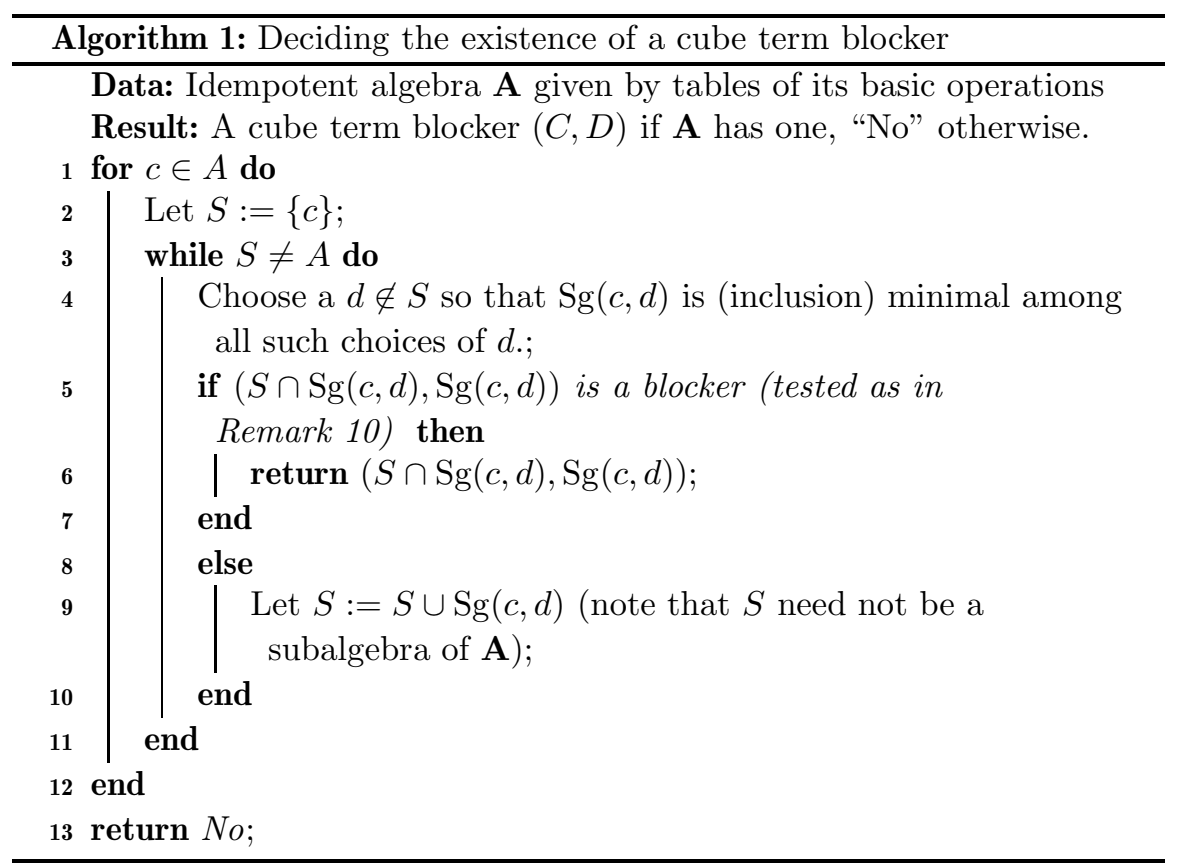

but no cube term of dimension 2: Consider $\mathbf{A}=(\{0,1\}, \wedge, \vee)$. This algebra is compatible with the binary elusive relation $\{(0,0),(0,1),(1,1)\}$ (elusive tuple $(1,0))$ and so does not have a cube term of dimension 2 (known as the Maltsev operation). However, $\mathbf{A}$ has a ternary near unanimity term and thus a cube term of dimension 3 .

\section{Deciding cube terms in the idempotent case}

In this section, we describe a polynomial time algorithm that decides whether the input idempotent algebra $\mathbf{A}$ has a cube term.. What is more, if $\mathbf{A}$ has no cube term, the algorithm will prove this by outputting a cube term blocker for $\mathbf{A}$.

The idea of Algorithm 1 is to look for an, in a sense, "minimal" cube term blocker $(C, D)$. If an idempotent algebra $\mathbf{A}$ contains a cube term blocker $(C, D)$, then we can try to make $C$ and $D$ smaller. Take $c \in C$ and $d \in D \backslash C$; it is easy to verify that the set $(C \cap \operatorname{Sg}(c, d), \operatorname{Sg}(c, d))$ will then also be a cube term blocker.

It is not too computationally expensive to guess $c, d \in A$ and we can efficiently test if a pair of subsets of $\mathbf{A}$ is a cube term blocker by looking at basic operations of $\mathbf{A}$ (see Remark 10); hence the only remaining issue is how to construct the right set $C \cap \operatorname{Sg}(c, d)$.

A rough outline of our method for constructing $C \cap \operatorname{Sg}(c, d)$ is as follows: We guess $c \in A$ and then grow a set $S$ as slowly as possible, starting with 
$S=\{c\}$. In each step we either find a cube term blocker of the form $(S \cap D, D)$, or we grow $S$, so after at most $|A|$ steps we reach $S=A$ and stop.

Theorem 23. Given an idempotent algebra A as input, Algorithm 1 will in a polynomial time either find a cube term blocker in $\mathbf{A}$ or correctly conclude that $\mathbf{A}$ has a cube term (and thus $\mathbf{A}$ contains no cube term blocker).

Proof. Examining the pseudo-code of Algorithm 1, it is obvious that the algorithm runs in polynomial time - in the RAM model of computation, the time complexity is $O\left(m|A|^{2}|\mathbf{A}|\right.$ ) (where $m$ is the maximum arity of a basic operation of $\mathbf{A}$ ). It remains to prove the correctness of the algorithm.

Since the algorithm tests to see if each potential output is a blocker (line 5), it follows that if Algorithm 1 outputs a pair of sets, then this pair is a blocker. It remains to show that the algorithm will find a blocker when $\mathbf{A}$ contains one.

To this end, assume that $(C, D)$ is a blocker in $\mathbf{A}$ such that $D$ is (inclusion) minimal. Let us fix some $c \in C$. We will now show that when this $c$ is chosen as the value of the variable $c$ in the for-loop (lines 1 12) of Algorithm 1] the algorithm will find a blocker.

The only way finding a blocker can fail for this particular $c$ is if the inner loop of the algorithm (steps 311) eventually adds all the elements of $A$ to $S$. The algorithm might find a blocker by "accident," in which case we are done. Assume that this does not happen and let us wait for the first time when on line 4 a $d \notin S$ is chosen such that $\operatorname{Sg}(c, d) \cap D \nsubseteq C$. In other words, we assume that $S \cap D \subseteq C$ and that $\operatorname{Sg}(c, d) \backslash S$ has a nonempty intersection with $D \backslash C$.

Recall that $D$ is a subuniverse of $\mathbf{A}$. Were $\operatorname{Sg}(c, d) \cap D$ smaller than $\operatorname{Sg}(c, d)$, we could choose an element $d^{\prime} \in(\operatorname{Sg}(c, d) \cap D) \backslash C$ instead of $d$ and get $d^{\prime} \notin S$ with a smaller $\operatorname{Sg}\left(c, d^{\prime}\right) \subseteq D$. Hence from the minimality of $\operatorname{Sg}(c, d)$ we see that $\operatorname{Sg}(c, d) \subseteq D$ and so $d$ lies in $D$. Since $\operatorname{Sg}(c, d) \nsubseteq C$, the element $d$ must lie in $D \backslash C$. Finally, the minimality of $D$ then gives us $\operatorname{Sg}(c, d)=D$.

We now claim that $S \cap D=C$. If we prove this, we will be done: The pair of sets $(S \cap \operatorname{Sg}(c, d), \operatorname{Sg}(c, d))=(S \cap D, D)=(C, D)$ is a blocker, meaning that, instead of adding $d$ to $S$, Algorithm 1 will reach line 6 and output $(C, D)$.

We prove $S \cap D=C$ by showing two inclusions. We have $S \cap D \subseteq C$ by assumption. To see $S \cap D \supseteq C$, consider what would happen if there was $a \in C \backslash(S \cap D)=C \backslash S$. Then $a \notin S$ and yet $\operatorname{Sg}(c, a) \subseteq C \subsetneq D=\operatorname{Sg}(c, d)$. Therefore, in step 4 the set $D=\operatorname{Sg}(c, d)$ was not minimal and $a$ should have been chosen instead of $d$. This contradiction concludes our proof.

Remark 24. As a side note, it turns out that modifying our algorithm to only look for "nice" blockers is tricky: For example it is an NP-complete problem to decide if, given an idempotent algebra $\mathbf{A}$ and an element $b \in A$, there exists a blocker $(C, D)$ such that $b \in D \backslash C[1$. 


\section{Cube terms in general algebras}

Let now $\mathbf{A}=\left(A ; f_{1}, \ldots, f_{\ell}\right)$ be an algebra that is not idempotent. Assuming that $\mathbf{A}$ has a cube term, what is the smallest dimension of a cube term that $\mathbf{A}$ has? It is easy to see that $\mathbf{A}$ has a cube term of dimension $n$ if and only if the idempotent reduct of $\mathbf{A}$ has an cube term of dimension $n$. We also know that the minimal dimension of a cube term is the same as the minimal arity of a near unanimity term - if $\mathbf{A}$ has a near unanimity, that is.

It turns out that we can recover a bit more from the idempotent case:

Lemma 25. Let $n \geq 2$ be an integer and $\mathbf{A}$ an algebra containing a cube term of dimension $n+1$, but no cube term of dimension $n$ (where $n$ is a positive integer). Then there exists an $n$-ary elusive relation compatible with $\mathbf{A}$.

Proof. Since A does not have a cube term of dimension $n$, neither does the idempotent reduct $\mathbf{A}_{i d m p}$ of $\mathbf{A}$. Therefore, there exist tuples $\mathbf{a}, \mathbf{b} \in A^{n}$ such that a does not lie in the subalgebra of $\mathbf{A}_{i d m p}$ generated by $\left\{\chi_{I}(\mathbf{a}, \mathbf{b}): I \neq \emptyset\right\}$.

Translating the last sentence from $\mathbf{A}_{i d m p}$ back to $\mathbf{A}$, we obtain that

$$
\operatorname{seq}(A) \mathbf{a} \notin \operatorname{Sg}_{\mathbf{A}|A|+n}\left(\left\{\operatorname{seq}(A) \chi_{I}(\mathbf{a}, \mathbf{b}): I \neq \emptyset\right\}\right) .
$$

Let now $\mathbf{q}$ be the shortest tuple of elements of $A$ for which

$$
\mathbf{q a} \notin \operatorname{Sg}_{\mathbf{A}|\mathbf{q}|+n}\left(\left\{\mathbf{q} \chi_{I}(\mathbf{a}, \mathbf{b}): I \neq \emptyset\right\}\right) .
$$

By the above reasoning, we have $|\mathbf{q}| \leq|A|$. We will show that in fact $|\mathbf{q}|=0$, proving the Lemma. Assume that $\mathbf{q}$ is of length at least one; we will show how this leads to a contradiction.

Let $\mathbf{q}=\mathbf{r} s$ for a suitable tuple $\mathbf{r}$ and $s \in A$. Denote by $E$ the relation $\operatorname{Sg}\left(\left\{\mathbf{q} \chi_{I}(\mathbf{a}, \mathbf{b}): I \neq \emptyset\right\}\right)$. Since we took $\mathbf{q}=\mathbf{r} s$ shortest possible, we must have

$$
\mathbf{r a} \in \operatorname{Sg}\left(\left\{\mathbf{r} \chi_{I}(\mathbf{a}, \mathbf{b}): I \neq \emptyset\right\}\right), \quad \mathbf{r} s \mathbf{a} \notin \operatorname{Sg}\left(\left\{\mathbf{r} s \chi_{I}(\mathbf{a}, \mathbf{b}): I \neq \emptyset\right\}\right)=E .
$$

We conclude that there exists $u \in A$ such that $\mathbf{r} u \mathbf{a} \in E$.

Now since $\mathbf{A}$ has a cube term of dimension $n+1$, it also has an edge term $t$ of dimension $n+1$ (by Theorem 4). We apply $t$ to the following matrix of tuples:

$$
\left(\begin{array}{cccccc}
\mathbf{r} & \mathbf{r} & \mathbf{r} & \cdots & \mathbf{r} & \mathbf{r} \\
s & s & s & \cdots & s & u \\
\chi_{\{1,2\}}(\mathbf{a}, \mathbf{b}) & \chi_{\{1\}}(\mathbf{a}, \mathbf{b}) & \chi_{\{2\}}(\mathbf{a}, \mathbf{b}) & \cdots & \chi_{\{n\}}(\mathbf{a}, \mathbf{b}) & \mathbf{a}
\end{array}\right)
$$

This matrix has $n+2$ columns, all of which are in $E$ (the tuples in the first $n+1$ columns are among the tuples that witness elusiveness of $E$ while the last tuple is $\mathbf{r} u \mathbf{a} \in E$ by the choice of $u$ ). Thus $t$ applied to the matrix outputs a member of $E$. However, using the identities for edge terms, one can easily verify that the output tuple is in fact $\mathbf{r} s \mathbf{a}$, a contradiction with $\mathbf{r} s \mathbf{a} \notin E$.

In the rest of this Section, let $R$ be an $n$-ary relation that is inclusion minimal among all $n$-ary elusive relations compatible with $\mathbf{A}$. Moreover, we 
order the coordinates of $R$ so that the two tuples that witness the elusiveness of $R$ are of the form

$$
\begin{aligned}
\mathbf{a} & =a_{1}^{\mathbf{n}_{1}} a_{2}^{\mathbf{n}_{2}} \ldots a_{k}^{\mathbf{n}_{\mathbf{k}}} \\
\mathbf{b} & =b_{1}^{\mathbf{n}_{1}} b_{2}^{\mathbf{n}_{2}} \ldots b_{k}^{\mathbf{n}_{\mathbf{k}}},
\end{aligned}
$$

with $\left(a_{i}, b_{i}\right) \neq\left(a_{j}, b_{j}\right)$ for $i \neq j$. Call such a pair elusive tuple of type $\left(n_{1}, n_{2}, \ldots, n_{k}\right)$.

The numbers $n_{1}, \ldots, n_{k}$ give us a partition of $n$ into intervals of consecutive integers. As before, we will call the members of this partition blocks. The $i$-th block, which we again denote by $B_{i}$, consists of the indices $B_{i}=$ $\left\{j \in \mathbb{N}: n_{1}+\cdots+n_{i-1}<j \leq n_{1}+\cdots+n_{i}\right\}$.

Definition 26. Assume that we have a fixed $\mathbf{A}, n_{1}, n_{2}, \ldots, n_{k} \in \mathbb{N}$, and $\mathbf{a}, \mathbf{b} \in$ $A^{n}$ are two tuples of type $\left(n_{1}, n_{2}, \ldots, n_{k}\right)$. Let $D_{1}, \ldots, D_{k}$ be such that there exists a term $t$ of $\mathbf{A}$ so that $D_{i}=t\left(\left\{a_{i}, b_{i}\right\},\left\{a_{i}, b_{i}\right\}, \ldots,\left\{a_{i}, b_{i}\right\}\right)$ for each $i$, and let $C_{i} \subseteq D_{i}$ for each $i$.

Then we define a blob of type $\left(n_{1}, \ldots, n_{k}\right)$, given by the sets $D_{1}, \ldots, D_{k}$ and $C_{1}, \ldots, C_{k}$, as

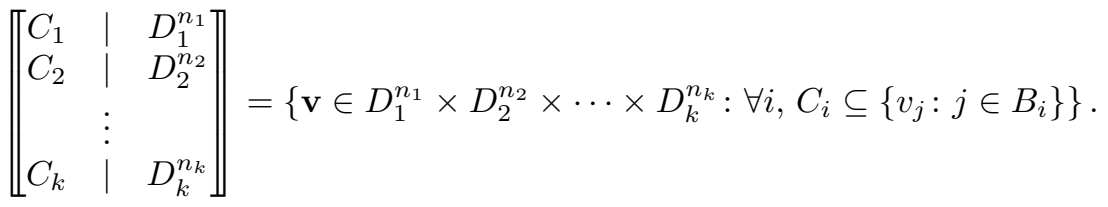

A union of a family of blobs of type $\left(n_{1}, n_{2}, \ldots, n_{k}\right)$ is called a sponge of type $\left(n_{1}, n_{2}, \ldots, n_{k}\right)$.

Sponges and blobs are distant relatives of chipped cubes (for example, one can write any chipped cube in the form of a sponge). We have shown that minimal compatible elusive relations are chipped cubes in the idempotent case; for general algebras, we want to show that the minimal compatible elusive relations are sponges (after a suitable reordering of coordinates). Before we do that, though, we need to obtain some tools.

Blobs and sponges carry with them information about blocks of coordinates, so we can talk about, say, the second block of coordinates of a blob $\Gamma$. We will be talking quite a bit about "the set of values appearing in a certain block of a tuple," so let us introduce a short name for this concept:

Definition 27. For $\mathbf{d} \in A^{k}$, we denote by $\operatorname{cont}(\mathbf{d})$ the content of $\mathbf{d}$, which is defined as the set of all elements of $A$ that appear in $\mathbf{d}$, i.e. $\operatorname{cont}\left(u_{1} u_{2} \ldots u_{k}\right)=$ $\left\{u_{1}, u_{2}, \ldots, u_{k}\right\}$. If $\mathbf{v} \in A^{n}$ is a tuple and we have a partition of $[n]$ into blocks $B_{1}, \ldots, B_{k}$ then the content of the $i$-th block of $\mathbf{v}$ is the set $\left\{v_{j}: j \in B_{i}\right\}$.

In the language of content, a blob is a set of $\mathbf{v} \in D_{1}^{n_{1}} \times D_{2}^{n_{2}} \times \cdots \times D_{k}^{n_{k}}$ such that for each $i=1, \ldots, k$, the content of the $i$-th block of $\mathbf{v}$ contains $C_{i}$.

It will also be useful to remap entries of tuples in a prescribed way.

Definition 28. For a map $\eta:[n] \rightarrow[n]$ and a tuple $\mathbf{a}=\left(a_{1}, \ldots, a_{n}\right) \in A^{n}$, define the $\eta$-image of $\mathbf{a}$ as $\mathbf{a}^{\eta}=\left(a_{\eta(1)}, a_{\eta(2)}, \ldots, a_{\eta(n)}\right)$. Define the $\eta$-image of 
$R \subseteq A^{n}$, denoted by $R^{\eta}$, as the set of $\eta$-images of all members of $R$. We say that $R$ is $\eta$-invariant if $R^{\eta} \subseteq R$.

Note that $\eta$ need not be a permutation. It is easy to see that if $\mathbf{b}_{\mathbf{1}}, \ldots, \mathbf{b}_{\mathbf{k}}$ are $n$-tuples then $\left(t\left(\mathbf{b}_{\mathbf{1}}, \ldots, \mathbf{b}_{\mathbf{k}}\right)\right)^{\eta}=t\left(\mathbf{b}_{\mathbf{1}}{ }^{\eta}, \ldots, \mathbf{b}_{\mathbf{k}}{ }^{\eta}\right)$. Because $\eta$-images and operations commute, $\eta$-images play nicely with subalgebras:

Observation 29. Let $\mathbf{A}$ be an algebra, $n \in \mathbb{N}, \eta:[n] \rightarrow[n], R, S \subseteq A^{n}$. Then

(a) If $R$ is compatible with $\mathbf{A}$, then $R^{\eta}$ is compatible with $\mathbf{A}$,

(b) if $R=\operatorname{Sg}(S)$, then $R$ is $\eta$-invariant if and only if $\mathbf{s}^{\eta} \in R$ for each $\mathbf{s} \in S$,

(c) if $S$ is a chipped cube, a sponge or a blob and $\eta$ is a permutation that preserves the blocks of $S$ (i.e. $\eta$ sends each block $B_{i}$ of $S$ to itself), then $S^{\eta}=S$.

(d) if $S$ is a chipped cube, $R=\operatorname{Sg}(S)$, and $\eta$ is a permutation of the coordinates of $S$ that preserves the blocks of $S$ then $R$ is $\eta$-invariant.

Proof. The first point follows in a straightforward way from the definition of a compatible relation. To prove the second point, realize that the $\eta$-image of $R$ is generated by $S^{\eta} \subseteq R$.

To see the third point, consider first the case when $S$ is a chipped cube or a blob. The membership of a tuple $\mathbf{d} \in A^{n}$ in $S$ only depends on the contents of all blocks of $\mathbf{d}$. But the content of the $i$-th block of $\mathbf{d}$ and $\mathbf{d}^{\eta}$ is the same for all $i$, therefore $S^{\eta}=S$. When $S$ is a sponge, $S$ is just a union of blobs of the same type - we can use the above argument for each blob separately and obtain $S^{\eta}=S$, too.

For the last point, we observe that $R^{\eta}=\operatorname{Sg}\left(S^{\eta}\right)$ because operations commute with $\eta$-images, and that $S^{\eta}=S$ by the previous point. Together, we get $R^{\eta}=\operatorname{Sg}\left(S^{\eta}\right)=\operatorname{Sg}(S)=R$.

The following observation follows directly from the definition of a blob:

Observation 30. Let $\Gamma$ be a blob and $\mathbf{r} \in A^{n}$. Let $\eta:[n] \rightarrow[n]$ be a mapping that sends each block of $\Gamma$ to itself. Assume moreover that the $i$-th block of $\mathbf{r}^{\eta}$ contains $C_{i}$ for each $i=1, \ldots, k$ (where $k$ is the number of blocks of $\Gamma$ ). Then $\mathbf{r} \in \Gamma$.

Observation 31. Let $\mathbf{a}, \mathbf{b} \in A^{n}$ be two tuples, $I \subseteq[n]$ and $\eta:[n] \rightarrow[n]$ a mapping. Then $\left(\chi_{I}(\mathbf{a}, \mathbf{b})\right)^{\eta}=\chi_{\eta^{-1}(I)}\left(\mathbf{a}^{\eta}, \mathbf{b}^{\eta}\right)$, where $\eta^{-1}(I)=\{j \in[n]: \eta(j) \in$ $I\}$.

Proof. Take $i \in[n]$. It follows from the definition of $\chi_{I}$ that the $i$-th entry of both $\left(\chi_{I}(\mathbf{a}, \mathbf{b})\right)^{\eta}$ and $\chi_{\eta^{-1}(I)}\left(\mathbf{a}^{\eta}, \mathbf{b}^{\eta}\right)$ is equal to $b_{\eta(i)}$ if $\eta(i) \in I$ and $a_{\eta(i)}$ otherwise.

It turns out that small elusive relations compatible with the algebra $\mathbf{A}$ are $\eta$-invariant exactly when $\eta$ is a permutation: 
Observation 32. Let $R=\operatorname{Sg}\left(\left\{\chi_{I}(\mathbf{a}, \mathbf{b}): I \neq \emptyset\right\}\right)$ be a relation such that $\mathbf{a} \notin R$, where

$$
\begin{aligned}
& \mathbf{a}=a_{1}^{\mathbf{n}_{1}} a_{2}^{\mathbf{n}_{2}} \ldots a_{k}^{\mathbf{n}_{\mathbf{k}}} \\
& \mathbf{b}=b_{1}^{\mathbf{n}_{1}} b_{2}^{\mathbf{n}_{2}} \ldots b_{k}^{\mathbf{n}_{\mathbf{k}}} .
\end{aligned}
$$

Let $\eta:[n] \rightarrow[n]$ preserve the blocks of $R$ (given by $n_{1}, n_{2}, \ldots, n_{k}$ ). Then $R^{\eta} \subseteq R$ if and only if $\eta$ is a permutation.

Proof. Note that since $\eta$ preserves the blocks of $R$, we have $\mathbf{a}^{\eta}=\mathbf{a}$ and $\mathbf{b}^{\eta}=\mathbf{b}$.

If $\eta$ is a permutation, then it sends the set of generators of $R$ to itself. Observation 29 then gives us $R^{\eta} \subseteq R$. On the other hand, assume that $\eta$ is not a permutation. Then $\eta$ is not onto; without loss of generality assume that 1 does not lie in the image of $\eta$. This gives us a contradiction, though:

$$
\left(\chi_{\{1\}}(\mathbf{a}, \mathbf{b})\right)^{\eta}=\chi_{\emptyset}\left(\mathbf{a}^{\eta}, \mathbf{b}^{\eta}\right)=\mathbf{a}^{\eta}=\mathbf{a} \notin R,
$$

where the first equality follows from Observation 31 and $\eta^{-1}(1)=\emptyset$. We see that $\eta$ sends the generator $\chi_{\{1\}}(\mathbf{a}, \mathbf{b})$ of $R$ outside of $R$, a failure of $\eta$ invariance of $R$.

Lemma 33. Let $\mathbf{A}$ be a finite algebra, and let $R$ be inclusion minimal among all elusive relations compatible with $\mathbf{A}$. Assume that $R=\operatorname{Sg}\left(\left\{\chi_{I}(\mathbf{a}, \mathbf{b}): I \neq\right.\right.$ $\emptyset\})$ and $\mathbf{a} \notin R$ for some tuples $\mathbf{a}, \mathbf{b}$ of type $\left(n_{1}, n_{2}, \ldots, n_{k}\right)$. Let $\mathbf{r}=w(M)$ where $w$ is a member of the clone of operations of $\mathbf{A}$ and $M$ is a matrix whose columns lie in $\left\{\chi_{I}(\mathbf{a}, \mathbf{b}): I \neq \emptyset\right\}$. Then $R$ contains the blob

$$
\Gamma=\llbracket \begin{array}{ccc}
C_{1} & \mid & D_{1}^{n_{1}} \\
C_{2} & \mid & D_{2}^{n_{2}} \\
& \vdots & \\
C_{k} & \mid & D_{k}^{n_{k}}
\end{array} \rrbracket
$$

where $D_{i}=w\left(\left\{a_{i}, b_{i}\right\}, \ldots,\left\{a_{i}, b_{i}\right\}\right)$ and $C_{i}=\left\{r_{j}: j \in B_{i}\right\}$.

Proof. Assume that $\Gamma \backslash R$ is nonempty. We shall show how this yields a contradiction. Choose a $\mathbf{d} \in \Gamma \backslash R$ and pick an $\eta:[n] \rightarrow[n]$ that preserves the blocks $B_{1}, \ldots, B_{k}$ and sends $\mathbf{d}$ to $\mathbf{r}$. (To construct such an $\eta$, let $\eta(j)$ for $j \in B_{i}$ be any $q \in B_{i}$ such that $d_{q}=r_{j}$.) Were $\eta$ a permutation, we would have $\mathbf{r}^{\eta^{-1}}=\mathbf{d}$. But $\eta^{-1}$ would then be a permutation that preserves blocks of coordinates, so $\mathbf{d} \in R^{\eta^{-1}} \subseteq R$, a contradiction with our choice of d. We conclude that $\eta$ is not a permutation. By Observation $32, R$ is not $\eta$-invariant.

In the rest of the proof, we shall show that $R^{\eta} \subseteq R$, which will be a contradiction.

Let $\mathbf{c}=c_{1}^{\mathbf{n}_{1}} c_{2}^{\mathbf{n}_{2}} \ldots c_{k}^{\mathbf{n}_{\mathbf{k}}}$ where $c_{i}=w\left(b_{i}, \ldots, b_{i}\right)$. We claim that for any nonempty $I \subseteq[n]$ we have $\chi_{I}(\mathbf{d}, \mathbf{c}) \in R$. We know that $\mathbf{d}=w(N)$ for a suitable matrix $N$ whose first $n_{1}$ rows contain only members of $\left\{a_{1}, b_{1}\right\}$, the next $n_{2}$ rows contain only members of $\left\{a_{2}, b_{2}\right\}$, etc. Now rewrite all rows of 
$N$ whose indices lie in $I$ to $b_{j}$ 's - call the new matrix $N^{\prime}$. Since $I \neq \emptyset$, each column of $N^{\prime}$ will contain a $b_{j}$, so columns of $N^{\prime}$ lie in $R$. It now remains to observe that $w\left(N^{\prime}\right)=\chi_{I}(\mathbf{d}, \mathbf{c}) \in R$.

Given that $\mathbf{d} \notin R$, but $\chi_{I}(\mathbf{d}, \mathbf{c}) \in R$ for any $I \neq \emptyset$, the tuple $\mathbf{d}$ is elusive for $R$. What is more, from the minimality of $R$ we get that $R=$ $\operatorname{Sg}\left(\left\{\chi_{I}(\mathbf{d}, \mathbf{c}): I \neq \emptyset\right\}\right)$.

To verify that $R^{\eta} \subseteq R$, it is therefore enough to show that $\chi_{I}(\mathbf{d}, \mathbf{c})^{\eta} \in R$ for each $I$. From Observation 31 and from $\mathbf{d}^{\eta}=\mathbf{r} \in R$ and $\mathbf{c}^{\eta}=\mathbf{c}$, we have

$$
\chi_{I}(\mathbf{d}, \mathbf{c})^{\eta}=\chi_{\eta^{-1}(I)}\left(\mathbf{d}^{\eta}, \mathbf{c}^{\eta}\right)=\chi_{\eta^{-1}(I)}(\mathbf{r}, \mathbf{c}) .
$$

However, since $\mathbf{r} \in D_{1}^{n_{1}} \times \cdots \times D_{k}^{n_{k}}$, we can rewrite any set of coordinates of $\mathbf{r}$ to coordinates of $\mathbf{c}$ and stay inside $R$ (this includes rewriting the empty set thanks to $\mathbf{r} \in R$ ). Therefore, $\chi_{I}(\mathbf{d}, \mathbf{c})^{\eta}=\chi_{\eta^{-1}(I)}(\mathbf{r}, \mathbf{c}) \in R$, making $R$ $\eta$-invariant, a contradiction.

Theorem 34. Let $\mathbf{A}$ be a finite algebra. Let $R$ be a relation that is inclusion minimal among $n$-ary elusive relations compatible with $\mathbf{A}$. Let the pair of tuples $\mathbf{a}, \mathbf{b}$ witness the elusiveness of $R$. Then we can reorder the coordinates of $R$ to get a sponge of type $\left(n_{1}, \ldots, n_{k}\right)$ where $k$ is the number of distinct pairs $\left(a_{i}, b_{i}\right)$ for $i=1, \ldots, n$.

Proof. Let us group the same pairs of entries of $\mathbf{a}, \mathbf{b}$ together. That is, we permute the coordinates $1, \ldots, n$ so that

$$
\begin{aligned}
& \mathbf{a}=a_{1}^{\mathbf{n}_{1}} a_{2}^{\mathbf{n}_{2}} \ldots a_{k}^{\mathbf{n}_{\mathbf{k}}} \\
& \mathbf{b}=b_{1}^{\mathbf{n}_{1}} b_{2}^{\mathbf{n}_{2}} \ldots b_{k}^{\mathbf{n}_{\mathbf{k}}},
\end{aligned}
$$

where $\left(a_{i}, b_{i}\right) \neq\left(a_{j}, b_{j}\right)$ for $i \neq j$. Observe that $k$ is then equal to the number of distinct pairs $\left(a_{i}, b_{i}\right)$, as required. Let us denote by $E$ the union of all blobs of type $\left(n_{1}, \ldots, n_{k}\right)$ that are contained in $R$. Our goal is to show that $E=R$.

Obviously, $E \subseteq R$, so all we need to show is that whenever $\mathbf{r} \in R$, then $\mathbf{r} \in \Gamma$ for some blob $\Gamma \subseteq R$. Consider any $\mathbf{r} \in R$. By the minimality of $R$, there is a term operation $w$ of $\mathbf{A}$ such that $\mathbf{r}=w\left(\chi_{I}(\mathbf{a}, \mathbf{b}): I \neq \emptyset\right)$. Applying Lemma 33. we get a blob $\Gamma$ such that $\mathbf{r} \in \Gamma \subseteq R$ - exactly what we need to show that $R=E$.

Lemma 35. Let $R$ be a relation inclusion minimal among all elusive relations compatible with $\mathbf{A}$. Assume moreover that $R$ is also a sponge of type $\left(n_{1}, n_{2}, \ldots, n_{k}\right)$. Let $m$ be the maximum arity of a basic operation of $\mathbf{A}$. If $\mathbf{A}$ has a cube term, then for each $i$ we have $n_{i}<|A| m$.

Proof. By the minimality of $R$ as an elusive compatible relation, we know that there are two tuples $\mathbf{a}=a_{1}^{\mathbf{n}_{1}} a_{2}^{\mathbf{n}_{2}} \ldots a_{k}^{\mathbf{n}_{\mathbf{k}}}$ and $\mathbf{b}=b_{1}^{\mathbf{n}_{1}} b_{1}^{\mathbf{n}_{2}} \ldots b_{k}^{\mathbf{n}_{\mathbf{k}}}$ so that $\mathbf{a} \notin R$ and $R=\operatorname{Sg}\left(\left\{\chi_{I}(\mathbf{a}, \mathbf{b}): I \neq \emptyset\right\}\right)$.

For each $\mathbf{r} \in R$, we consider all the blobs of type $\left(n_{1}, \ldots, n_{k}\right)$ that the conclusion of Lemma 33 places inside $R$ (cf. proof of Theorem 34). Such blobs 
cover $R$, so we have (for a suitable set $L$, and appropriate $C_{i, \ell}$ 's and $D_{i, \ell}$ 's):

$$
R=\bigcup_{\ell \in L} \llbracket \begin{array}{lll}
C_{1, \ell} & \mid & D_{1, \ell}^{n_{1}} \\
C_{2, \ell} & \mid & D_{2, \ell}^{n_{2}} \\
& \vdots & \\
C_{k, \ell} & \mid & D_{k, \ell}^{n_{k}}
\end{array} \rrbracket
$$

Assume for a contradiction (and without loss of generality) that $n_{1} \geq$ $m|A|$. We shall show that $\mathbf{A}$ does not have a cube term. For $s \in \mathbb{N}$ we define the relation $R^{\star s}$ as basically " $R$ whose first block is extended by $s$ entries":

$$
R^{\star s}=\bigcup_{\ell \in L} \llbracket \begin{array}{ccc}
C_{1, \ell} & \mid & D_{1, \ell}^{n_{1}+s} \\
C_{2, \ell} & D_{2, \ell}^{n_{2}} \\
& \vdots & \\
C_{k, \ell} & \mid & D_{k, \ell}^{n_{k}}
\end{array} \rrbracket .
$$

Let $B_{1}^{\star s}, B_{2}^{\star s}, \ldots$ be the blocks of $R^{\star s}$; we denote the tuples $a_{1}^{\mathbf{n}_{1}+\mathbf{s}} a_{2}^{\mathbf{n}_{2}} \ldots$ and $b_{1}^{\mathbf{n}_{1}+\mathbf{s}} b_{2}^{\mathbf{n}_{2}} \ldots$ by $\mathbf{a}^{\star s}$ and $\mathbf{b}^{\star s}$, respectively.

It is easy to see that $R^{\star s}$ contains $\chi_{I}\left(\mathbf{a}^{\star s}, \mathbf{b}^{\star s}\right)$ for each nonempty $I \subseteq$ $[n+s]$, but $\mathbf{a}^{\star s} \notin R^{\star s}$, so $R^{\star s}$ is an elusive relation. If we can now show that each $R^{\star s}$ is also compatible with $\mathbf{A}$, we will have a family of arbitrarily large elusive relations compatible with $\mathbf{A}$. Therefore, $\mathbf{A}$ will have no cube term by Lemma 14.

Take any basic operation $t$ of $\mathbf{A}$ of arity $r \leq m$ and let $\mathbf{c}_{\mathbf{1}}, \ldots, \mathbf{c}_{\mathbf{r}} \in R^{\star s}$. We want to show that then also $t\left(\mathbf{c}_{\mathbf{1}}, \mathbf{c}_{\mathbf{2}}, \ldots, \mathbf{c}_{\mathbf{r}}\right) \in R^{\star s}$. To simplify notation, we will assume that each $\mathbf{c}_{\mathbf{i}}$ belongs to the $i$-th blob from $L$ (this is without loss of generality, as we can reorder blobs and even take several copies of the same blob without changing $R^{\star s}$ ).

Let us now examine the content of the first block of entries of $\mathbf{c}_{\mathbf{1}}, \ldots, \mathbf{c}_{\mathbf{r}}$. Let $\mathbf{d}_{\mathbf{i}}=\left(\mathbf{c}_{\mathbf{i}}\right)_{\left[1, n_{1}+s\right]}$ for $i=1, \ldots, r$. Then $\operatorname{cont}\left(\mathbf{d}_{\mathbf{i}}\right)$ has size at most $|A|$ for each $i=1, \ldots, r$ and thus there are at most $r|A|$ indices in $B_{1}^{\star s}$ that witness the content of all $\mathbf{d}_{\mathbf{1}}, \ldots, \mathbf{d}_{\mathbf{r}}$. Since $n_{1} \geq r|A|$ and $R^{\star s}$ is invariant under permuting the first block, we can assume that the complete content of all $\mathbf{d}_{\mathbf{i}}$ 's appears in the last $n_{1}$ entries of $B_{1}^{\star s}$. That is, for each $i=1, \ldots, r$ we have

$$
\operatorname{cont}\left(\left(\mathbf{c}_{\mathbf{i}}\right)_{\left[1, s+n_{1}\right]}\right)=\operatorname{cont}\left(\left(\mathbf{c}_{\mathbf{i}}\right)_{\left[s+1, s+n_{1}\right]}\right) .
$$

Let now $\mathbf{e}_{\mathbf{i}}=\left(\mathbf{c}_{\mathbf{i}}\right)_{[s+1, n]}$, i.e. $\mathbf{e}_{\mathbf{i}}$ is obtained from $\mathbf{c}_{\mathbf{i}}$ by cutting away the first $s$ entries. Since the contents of the first blocks remain the same, we see

$$
\mathbf{e}_{\mathbf{i}} \in \llbracket \llbracket \begin{array}{lll}
C_{1, i} & \mid & D_{1, i}^{n_{1}} \\
C_{2, i} & \mid & D_{2, i}^{n_{2}} \\
& \vdots & \\
C_{k, i} & \mid & D_{k, i}^{n_{k}}
\end{array} \|,
$$

so $\mathbf{e}_{\mathbf{i}} \in R$. By the definition of $R$, for each $i$ there exists a $\left(2^{n}-1\right)$-ary operation $u_{i}$ in the clone of $\mathbf{A}$ such that $\mathbf{e}_{\mathbf{i}}=u_{i}\left(\chi_{I}(\mathbf{a}, \mathbf{b}): I \neq \emptyset\right)$. 
Therefore, $t\left(\mathbf{e}_{\mathbf{1}}, \ldots, \mathbf{e}_{\mathbf{r}}\right) \in R$. Denote by $w$ the $r\left(2^{n}-1\right)$-ary term $t \circ$ $\left(u_{1}, u_{2}, \ldots, u_{r}\right)$ (where each $u_{i}$ has its own distinct variable set). Let $M$ be the $n \times r\left(2^{n}-1\right)$ matrix that consists of $r$ copies of $\left(\chi_{I}(\mathbf{a}, \mathbf{b}): I \neq \emptyset\right)$ arranged next to each other. It is easy to verify that $w(M)=t\left(\mathbf{e}_{\mathbf{1}}, \ldots, \mathbf{e}_{\mathbf{k}}\right)$. Using Lemma 33 with the term $w$ and tuple $w(M)$, we obtain

$$
t\left(\mathbf{e}_{1}, \ldots, \mathbf{e}_{\mathbf{r}}\right) \in \llbracket \llbracket \begin{array}{ccc}
C_{1} & \mid & D_{1}^{n_{1}} \\
C_{2} & \mid & D_{2}^{n_{2}} \\
& \vdots & \\
C_{k} & \mid & D_{k}^{n_{k}}
\end{array} \| \subseteq R,
$$

where $C_{i}$ is the content of the $i$-th block of $w(M)$ and we let $D_{i}$ to be equal to $w\left(\left\{a_{i}, b_{i}\right\}, \ldots,\left\{a_{i}, b_{i}\right\}\right)$. Since the tuple $t\left(\mathbf{e}_{\mathbf{1}}, \ldots, \mathbf{e}_{\mathbf{r}}\right)$ is a suffix of $t\left(\mathbf{c}_{\mathbf{1}}, \ldots, \mathbf{c}_{\mathbf{r}}\right)$, we only need to verify that $t\left(\left(\mathbf{c}_{1}\right)_{j}, \ldots,\left(\mathbf{c}_{\mathbf{r}}\right)_{j}\right) \in D_{1}$ for each $j=1, \ldots, s$ to obtain

finishing the proof.

$$
t\left(\mathbf{c}_{\mathbf{1}}, \ldots, \mathbf{c}_{\mathbf{r}}\right) \in \llbracket \llbracket \begin{array}{ccc}
C_{1} & \mid & D_{1}^{n_{1}+s} \\
C_{2} & \mid & D_{2}^{n_{2}} \\
& \vdots & \\
C_{k} & \mid & D_{k}^{n_{k}}
\end{array} \| \subseteq R^{\star s},
$$

Pick a $j \in\{1, \ldots, s\}$. We know that $\left(\mathbf{c}_{1}\right)_{j} \in \operatorname{cont}\left(\mathbf{e}_{1}\right)_{\left[1, n_{1}\right]}$ and so

$$
\left(\mathbf{c}_{1}\right)_{j} \in u_{1}\left(\left\{a_{1}, b_{1}\right\}, \ldots,\left\{a_{1}, b_{1}\right\}\right) .
$$

We can do the same thing with $\left(\mathbf{c}_{2}\right)_{j},\left(\mathbf{c}_{\boldsymbol{3}}\right)_{j}$ and so on, getting

$$
\begin{aligned}
t\left(\left(\mathbf{c}_{1}\right)_{j}, \ldots,\left(\mathbf{c}_{\mathbf{r}}\right)_{j}\right) & \in t\left(u_{1}\left(\left\{a_{1}, b_{1}\right\}, \ldots\right), \ldots, u_{r}\left(\left\{a_{1}, b_{1}\right\}, \ldots\right)\right) \\
& \in w\left(\left\{a_{1}, b_{1}\right\},\left\{a_{1}, b_{1}\right\}, \ldots\right)=D_{1},
\end{aligned}
$$

which is exactly what we needed.

Theorem 36. Let $\mathbf{A}$ be a finite algebra with the universe $\{1,2, \ldots,|A|\}$. Let $m$ be the maximal arity of a basic operation in $\mathbf{A}$. Then $\mathbf{A}$ has a cube term if and only if $\mathbf{A}$ has a cube term of dimension at most $|A|^{3} \mathrm{~m}$.

Proof. The nontrivial implication is " $\Rightarrow$." Observe that the theorem is true (but not very interesting) when $|A|=1$ or $m=1$. Thus we let $|A|^{3} m>2$.

Assume that $\mathbf{A}$ has a cube term. Using Lemma 25, it is enough to show that every elusive relation compatible with $\mathbf{A}$ has arity less than $|A|^{3} \mathrm{~m}$.

Let $R$ be an $n$-ary elusive relation compatible with $\mathbf{A}$. By taking $R$ inclusion minimal and applying Theorem 34, we can assume without loss of generality that $R$ is a sponge of type $\left(n_{1}, \ldots, n_{k}\right)$ where $n_{1}+n_{2}+\cdots+n_{k}=n$ and $k \leq|A|^{2}$.

By Lemma 35 we have $n_{i}<|A| m$. Therefore

$$
n=\sum_{i=1}^{k} n_{i}<k|A| m \leq|A|^{2}|A| m=|A|^{3} m,
$$

giving us $n<|A|^{3} m$ as was needed. 


\section{Deciding cube and near unanimity terms is in EXPTIME}

We conclude our paper with two algorithmic corollaries of Theorem 36.

Corollary 37. The problem of deciding if a given finite algebra (given by its tables of basic operations) has a cube term is in EXPTIME.

Proof. We present an EXPTIME algorithm for the problem. Let $d=|A|^{3} m$, where $m$ is the maximum arity of a basic operation of $\mathbf{A}$. For each pair $a, b \in$ $A$, consider the subuniverse $R_{a, b}$ of $\mathbf{A}^{|A|+m|A|^{3}}$ generated by $\operatorname{seq}(A) \chi_{I}\left(a^{\mathbf{d}}, b^{\mathbf{d}}\right)$ for all $I \neq \emptyset$.

If $R_{a, b}$ does not contain the tuple seq $(A) a^{\mathbf{d}}$, then $\mathbf{A}$ has no $d$-dimensional cube term, and so no cube term at all by Theorem 36 . On the other hand, if $\mathbf{A}$ has no cube term, then let $\mathbf{A}_{i d m p}$ be the idempotent reduct of $\mathbf{A}$. We see by Proposition 8 that $\mathbf{A}_{i d m p}$ has a cube term blocker $(C, D)$. Pick $b \in C$ and $a \in D \backslash C$; from the definition of a blocker it follows that the subuniverse of $\mathbf{A}_{i d m p}^{d}$ generated by $\chi_{I}\left(a^{\mathbf{d}}, b^{\mathbf{d}}\right)$ for $I \neq \emptyset$ does not contain $a^{\mathbf{d}}$. Translating this back to $\mathbf{A}$, we obtain that $R_{a, b}$ does not contain $a^{\mathbf{d}}$, finishing the proof of correctness of our algorithm.

If $\mathbf{A}$ has $k$ basic operations of arity at most $m$, then $|\mathbf{A}|=O\left(k|A|^{m}\right)$ and generating each $R_{a, b}$ takes time $O\left(m k\left(|A|^{|A|+m|A|^{3}}\right)^{m}\right)$. Assume for simplicity that $k$ is much less than $2^{|A|}$. The time estimate for generating $R_{a, b}$ then becomes $2^{O\left(m^{2}|A|^{3} \log |A|\right)}$. Since there are only $|A|^{2}=2^{O(\log |A|)}$ many possible choices of $a, b$, the total running time of our algorithm will also be $2^{O\left(m^{2}|A|^{3} \log |A|\right)}$, placing the problem into the EXPTIME complexity class.

The algorithm above can be made a bit faster, but only at the cost of making it more complicated (by suitably generalizing the notion of cube term blocker). Even the best algorithm known to us still needs exponential time in the worst case. Since building up the machinery for generalized cube term blockers would take up several more pages and the complexity theoretic payoff is small, we have decided to present only the straightforward algorithm here.

As noted in the Introduction, an algebra has a near unanimity term if and only if it has a cube term and generates a congruence distributive variety. Deciding if a given algebra generates a congruence distributive variety lies in EXPTIME (in fact, it is EXPTIME-complete [7). Therefore, it follows from Corollary 37 that deciding if an algebra has a near unanimity term is in EXPTIME.

Corollary 38. The problem of deciding if a given finite algebra (given by tables of its basic operations) has a near unanimity term is in EXPTIME. 


\section{Conclusions}

We have shown that there are strong conditions that limit the way in which a finite algebra with finitely many basic operations can have a cube term. However, there are still open problems associated with this topic.

First of all, given a signature and a finite algebra $\mathbf{A}$ of this signature with a cube term, what is the minimal dimension of a cube term in the clone of $\mathbf{A}$ ? The construction for the idempotent case gives us a tight lower bound on the minimal dimension of cube terms. It is possible to do some minor improvements upon the $m|A|^{3}$ upper bound we have presented, but it remains open if there are large algebras with minimal cube term dimension $\Omega\left(m|A|^{3}\right)$.

It is often the case that various Maltsev conditions that are polynomial time decidable for idempotent algebras turn out to be hard for general algebras, see [7]. We conjecture that one can not do much better than the algorithm presented in Section 7 i.e. that the problem of deciding whether a given algebra has a cube term is, like the decision problems for many other Maltsev conditions, EXPTIME-complete.

\section{References}

[1] Barto, L., Kazda, A.: Deciding absorption. International Journal of Algebra and Computation 26(5), 1033-1060 (2016). DOI 10.1142/S0218196716500430. URL https://doi .org/10.1142/S0218196716500430

[2] Bergman, C.: Universal Algebra: Fundamentals and Selected Topics, 1st edn. Chapman \& Hall/CRC Press, Boca Raton and New York and Abingdon (2011)

[3] Berman, J., Idziak, P., Markovic, P., McKenzie, R., Valeriote, M., Willard, R.: Varieties with few subalgebras of powers. Transactions of the American Mathematical Society 362, 1445-1473 (2010). DOI 10.1090/S0002-9947-09-04874-0

[4] Bodnarchuk, V., Kaluzhnin, L., Kotov, V., Romov, B.: Galois theory for Post algebras. I. Cybernetics 5(3), 243-252 (1969). DOI 10.1007/BF01070906. URL http://dx.doi.org/10.1007/BF01070906

[5] Burris, S., Sankappanavar, H.P.: A Course in Universal Algebra, graduate texts in mathematics edn. Springer, New York (2012). URL http://www.math.uwaterloo.ca/ snburris/htdocs/ualg.html The Millenium Edition (electronic book)

[6] Freese, R., Kiss, E., Valeriote, M.: Universal Algebra Calculator (2011). Available at: www uacalc.org

[7] Freese, R., Valeriote, M.: On the complexity of some Maltsev conditions. International Journal of Algebra and Computation 19(01), 41-77 (2009). DOI 10.1142/S0218196709004956. URL https://www . worldscientific.com/doi/abs/10.1142/S0218196709004956

[8] Geiger, D.: Closed systems of functions and predicates. Pacific Journal of Mathematics. 27(1), 95-100 (1968)

[9] Horowitz, J.: Computational complexity of various Mal'cev conditions. International Journal of Algebra and Computation 
23(06), 1521-1531 (2013). DOI 10.1142/S0218196713500343. URL https://doi.org/10.1142/S0218196713500343

[10] Idziak, P., Marković, P., McKenzie, R., Valeriote, M., Willard, R.: Tractability and learnability arising from algebras with few subpowers. SIAM J. Comput. 39(7), 3023-3037 (2010). DOI 10.1137/090775646. URL http://dx.doi.org/10.1137/090775646

[11] Kearnes, K.A., Szendrei, A.: Clones of algebras with parallelogram terms. International Journal of Algebra and Computation 22(01), 1250,005 (2012). DOI 10.1142/S0218196711006716. URL http://www.worldscientific.com/doi/abs/10.1142/S0218196711006716

[12] Kearnes, K.A., Szendrei, Á.: Cube term blockers without finiteness. Algebra universalis 78(4), 437-459 (2017). DOI 10.1007/s00012-017-0476-6. URL https://doi.org/10.1007/s00012-017-0476-6

[13] Lindner, C.C., Rodger, C.A.: Design Theory, 2nd edn. Chapman \& Hall/CRC Press, Roca Baton, London, New York (2008)

[14] Marković, P., Maróti, M., McKenzie, R.: Finitely related clones and algebras with cube terms. Order 29(2), 345-359 (2012). DOI 10.1007/ s11083-011-9232-2. URL http://dx.doi.org/10.1007/s11083-011-9232-2

[15] Maróti, M.: The existence of a near-unanimity term in a finite algebra is decidable. The Journal of Symbolic Logic 74, 1001-1014 (2009). DOI 10.2178/jsl/1245158096. URL http://journals.cambridge.org/article_S0022481200003418

[16] Sequeira, L.: Near-unanimity is decomposable. Algebra Universalis $\mathbf{5 0}(2), \quad 157-164$ (2003). DOI 10.1007/s00012-003-1830-4. URL https://doi.org/10.1007/s00012-003-1830-4

[17] Zhuk, D.N.: Key (critical) relations preserved by a weak near-unanimity function. Algebra universalis $\mathbf{7 7}(2), \quad 191-235$ (2017). DOI 10.1007/ s00012-017-0426-3. URL https://doi.org/10.1007/s00012-017-0426-3

Alexandr Kazda

Department of Algebra, Charles University

Sokolovská 83, 18600 Praha 8, Czech Republic

ORCID 0000-0002-7338-037X

e-mail: alex.kazda@gmail.com

Dmitriy Zhuk

Department of Mathematics and Mechanics, Moscow State University

Moscow, Russia

e-mail: zhuk.dmitriy@gmail.com 